\title{
Commensal Propionibacterium strain UF1 mitigates intestinal inflammation via Th17 cell regulation
}

\author{
Natacha Colliou, ${ }^{1,2}$ Yong Ge, ${ }^{1,2}$ Bikash Sahay, ${ }^{1}$ Minghao Gong, ${ }^{1,2}$ Mojgan Zadeh, ${ }^{1,2}$ Jennifer L. Owen, ${ }^{3}$ Josef Neu, ${ }^{4}$ \\ William G. Farmerie, ${ }^{5}$ Francis Alonzo III, ${ }^{6}$ Ken Liu, ${ }^{7}$ Dean P. Jones, ${ }^{7}$ Shuzhao Li, ${ }^{7}$ and Mansour Mohamadzadeh ${ }^{1,2}$ \\ 'Department of Infectious Diseases and Immunology, 'Division of Castroenterology, Hepatology \& Nutrition, Department of Medicine, ${ }^{3}$ Department of Physiological Sciences, ${ }^{4}$ Division of Neonatology, \\ Department of Pediatrics, and 5 Interdisciplinary Center for Biotechnology Research, University of Florida, Gainesville, Florida, USA. ${ }^{6}$ Department of Microbiology and Immunology, Loyola University Chicago, \\ Maywood, Illinois, USA. `Division of Pulmonary, Allergy, Critical Care and Sleep Medicine, Department of Medicine, Emory University School of Medicine, Atlanta, Georgia, USA.
}

\begin{abstract}
Consumption of human breast milk (HBM) attenuates the incidence of necrotizing enterocolitis (NEC), which remains a leading and intractable cause of mortality in preterm infants. Here, we report that this diminution correlates with alterations in the gut microbiota, particularly enrichment of Propionibacterium species. Transfaunation of microbiota from HBM-fed preterm infants or a newly identified and cultured Propionibacterium strain, P. UF1, to germfree mice conferred protection against pathogen infection and correlated with profound increases in intestinal Th17 cells. The induction of Th17 cells was dependent on bacterial dihydrolipoamide acetyltransferase (DlaT), a major protein expressed on the P. UF1 surface layer (S-layer). Binding of P. UF1 to its cognate receptor, SICNR1, on dendritic cells resulted in the regulation of intestinal phagocytes. Importantly, transfer of P. UF1 profoundly mitigated induced NEC-like injury in neonatal mice. Together, these results mechanistically elucidate the protective effects of HBM and P. UF1-induced immunoregulation, which safeguard against proinflammatory diseases, including NEC.
\end{abstract}

\section{Introduction}

Despite major advancements in medicine, the rate of preterm birth has not decreased and remains one of the leading global causes of infant mortality (1). Maternal pathogen-induced inflammation potentially contributes to both preterm birth and fetal inflammatory responses (2). This detrimental inflammation results in significant deterioration of the microbial consortium of the neonatal gut (3) that skews immunity toward proinflammatory responses, possibly resulting in necrotizing enterocolitis (NEC), a potentially fatal inflammatory bowel necrosis primarily affecting preterm infants with risk factors (e.g., intestinal injury) (4). While the multifactorial mechanisms involved in NEC and potential therapeutic approaches remain elusive (5), the need for novel therapeutic interventions that redirect inflammation and reduce disease severity, leading to its regression, is urgent (6). Maintaining gut homeostasis to swiftly resolve gastrointestinal infections, which otherwise induce deleterious signals, is critically important and highly dependent on the fitness of host regulatory mechanisms. These mechanisms are dictated by critical nutrients in human breast milk (HBM) and the mother's and infant's microbiomes (7), which together make up a pivotal triad intricately involved in infant intestinal immunity (8). Errors in these mechanisms may increase pathologic signals, resulting in dysfunctional gut homeostasis, potentially leading to NEC.

Authorship note: N. Colliou and Y. Ge contributed equally to this work. Conflict of interest: M. Mohamadzadeh and B. Sahay are the inventors of P. UF1 (US provisional application no. US2016/032096).

Submitted: May 26, 2017; Accepted: August 2, 2017.

Reference information: / Clin Invest. 2017;127(11):3970-3986.

https://doi.org/10.1172/JCI95376.
Thus far, it has been postulated that HBM not only increases beneficial bacteria within balanced gut microbiota, but also promotes host immune regulation and reduces the risk of NEC in preterm infants (9-12). However, further mechanistic insights are required to better understand the immune regulation orchestrated by HBM, the beneficial bacteria within, and critical induced metabolites, all of which may control T cell responses (e.g., Th17 cells) intricately involved in mucosal protection and the pathogenesis of proinflammatory diseases (13). This includes NEC, in which the recruitment of inflammatory cells is robustly increased, while functional Tregs are significantly decreased (14). Thus, if severe induced inflammation leads to NEC, its redirection by novel therapeutic approaches involving beneficial commensals, including Propionibacterium (P.) University of Florida 1 (UF1) (P. UF1), may enhance proinflammatory NEC regression.

\section{Results}

Characterization of P. UF1 bacterium. Focusing on the status of the microbiota of 2 discrete cohorts, we demonstrate that the composition and diversity of the gut microbiota from human breast milkfed (HBMF) preterm infants $(n=20)$ were distinct from those of formula-fed $(\mathrm{FF})$ preterm infants $(n=20)$. Microbiota analyses of these 2 cohorts revealed major differences in the Shannon diversity, which estimates the total number of operational taxonomic units existing in the given bacterial community, and the Pielou's evenness index, which demonstrates how evenly the individuals in the community are distributed over different operational taxonomic units $(P<0.01)$ (Figure $1 \mathrm{~A})$. Together, the microbial evenness and diversity were found to be significantly increased in the HBMF cohort's microbiota, while a trend toward an increase in 
A

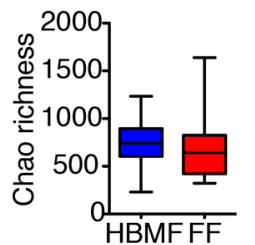

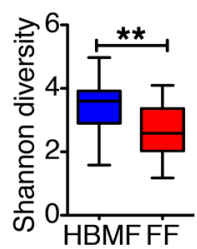

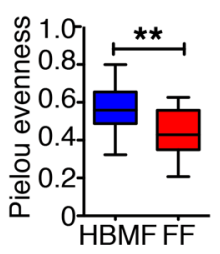

B

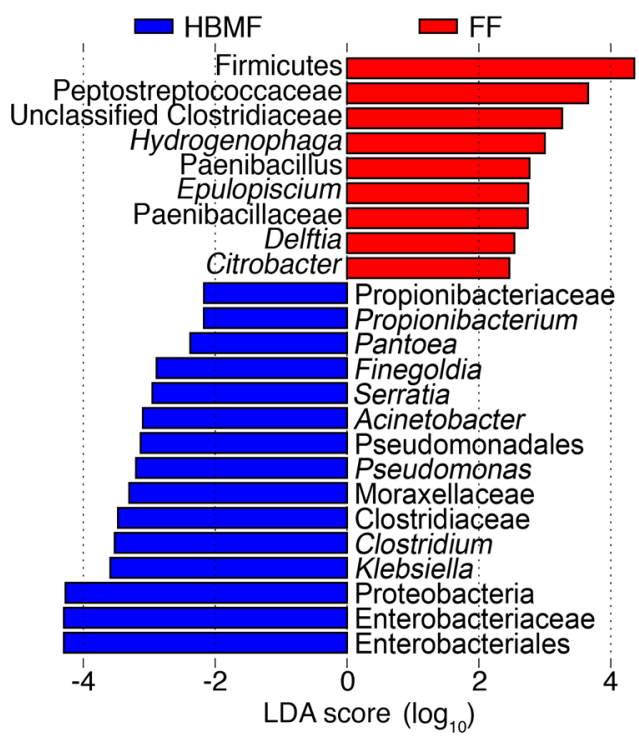

C

$\square$ Actinobacteria $\square$ Bacteroidetes $\square$ Firmicutes $\square$ Proteobacteria $\square$ Others
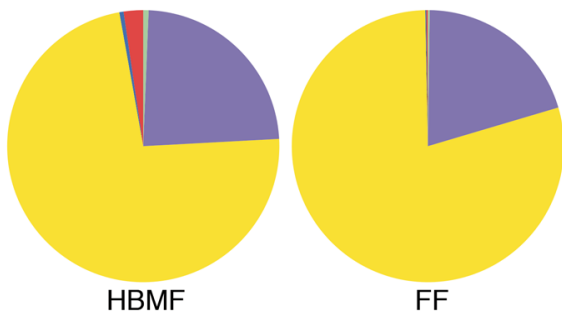

D
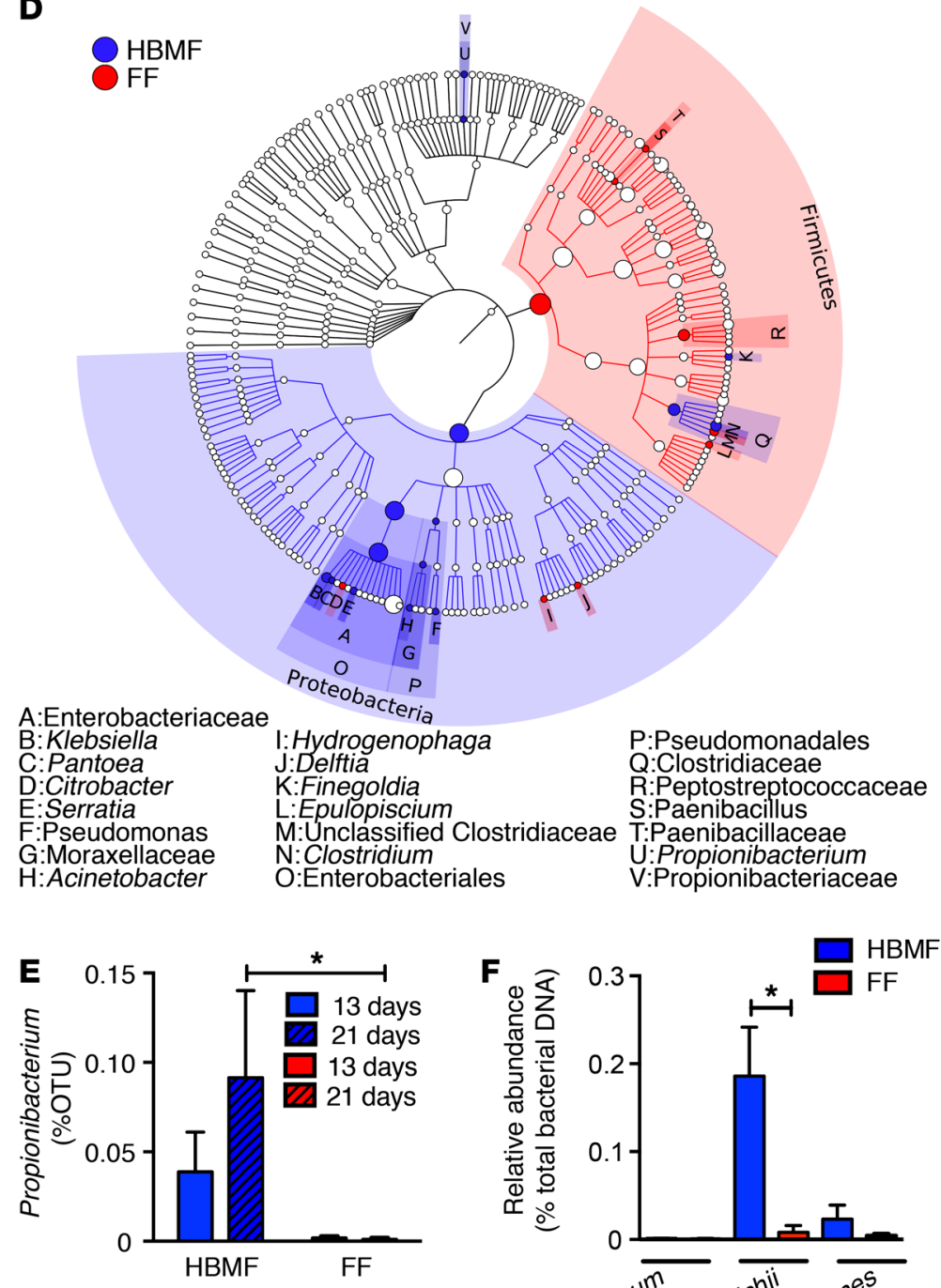

Figure 1. Abundance of Propionibacterium in the fecal samples of HBMF preterm infants. Fecal samples were collected from HBMF ( $n=20$ ) and FF preterm infants $(n=20)$, and microbiota composition was analyzed by 165 rDNA sequencing. (A) Summary box plots of Chao richness, Shannon diversity, and Pielou's evenness indices derived from analyses of fecal samples of HBMF (blue) and FF (red) preterm infants on day $13 \pm 2$ to 3 . (B) Linear discriminant analysis (LDA) of taxons between HBMF and FF preterm infants' microbiota on day $13 \pm 2$ to 3 . Taxa enriched in HBMF preterm infants' microbiota have a negative score (blue), and taxa enriched in FF preterm infants' microbiota have a positive score (red). Only taxa with an absolute value of LDA score of more than 2 are shown. (C) Phylum structure of the abundant bacteria in fecal samples of HBMF and FF preterm infants on days $21 \pm 3$. (D) Taxonomic cladogram of HBMF versus FF preterm infants' bacterial fecal samples on day $21 \pm 3$ (blue, HBMF-enriched taxa; red, FF-enriched taxa). (E) Percentage of operational taxonomic units (OTUs) of Propionibacteria in the fecal samples of HBMF and FF preterm infants by day $13 \pm 2$ to 3 and day $21 \pm 3$. (F) Relative abundance of different Propionibacteria (e.g., $P$. freudenreichii) in fecal samples of HBMF and FF preterm infants. Error bars indicate mean \pm SEM. ${ }^{*} P<0.05 ;{ }^{* *} P<0.01,2$-tailed unpaired $t$ test (A, E, and $\mathbf{F}$ ). Kruskal-Wallis test (B and $\left.\mathbf{D}\right)$.

bacterial richness was also observed (Figure 1A). Moreover, microbiota compositions between the HBMF and FF preterm infants were principally different in the Actinobacteria phylum (e.g., Propionibacteriaceae), among other bacteria, including Bacteriodetes, Firmicutes, and Proteobacteria (Figure 1, B-D). Thus, the focus was drawn to key species of this Actinobacteria phylum, particularly Propionibacterium, which contributed heavily to these differences (Figure 1, B-D). Importantly, the proportion of Propionibacterium, including $P$. freudenreichii, in the microbiota of the HBMF preterm infants was significantly increased throughout days 13 to 21 after birth, while these bacteria were poorly detected in the FF preterm infants' fecal samples (Figure 1, E and F). Subsequently, Propionibacterium was isolated from HBMF preterm infants' fecal samples using carbohydrate separation and cultured optimally in de Man, Rogosa, and Sharpe (MRS) broths and lactate.

The first draft genome-sequence analyses of one of these newly identified Propionibacterium strains, tentatively designated P. UF1, exhibited 90\% identity to known Propionibacterium species, including $P$. freudenreichii subsp. freudenreichii DSM $20271^{\mathrm{T}}$ and subsp. shermanii CIRM-BIA1 (Figure 2A). The genome of 
A

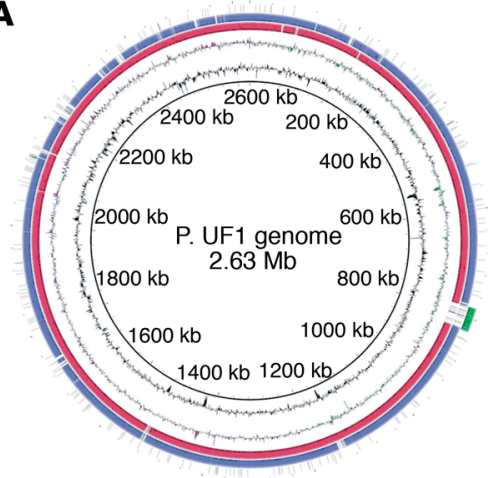

B

$\begin{array}{cc}\text { Subsystem } & \text { Subsystem category } \\ \text { coverage } & \text { distribution }\end{array}$

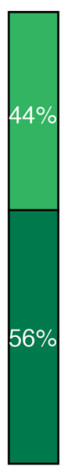

Subsystem feature counts

Cell Wall and Capsule (57)

Virulence, Disease and Defense (46)

Potassium Metabolism (9)

Miscellaneous (19)

Membrane Transport (45)

- Iron Acquisition and Metabolism (5)

RNA Metabolism (68)

Nucleosides and Nucleotides (60)

Protein Metabolism (202)

Cell Division and Cell Cycle (22)

Regulation and Cell signaling (8)

DNA Metabolism (95)

Fatty Acids, Lipids, and Isoprenoids (68)

Nitrogen Metabolism (9)

Dormancy and Sporulation (1)

Respiration (56)

Stress Response (56)

Metabolism of Aromatic Compounds (3)

Amino Acids and Derivatives (228)

Sulfur Metabolism (10)

Phosphorus Metabolism (26)

Carbohydrates (239)

Cofactors, Vitamins, Prosthetic Groups, Pigments (194)

Phages, Prophages, Transposable elements, Plasmids (5)

$\mathbf{E}$
C
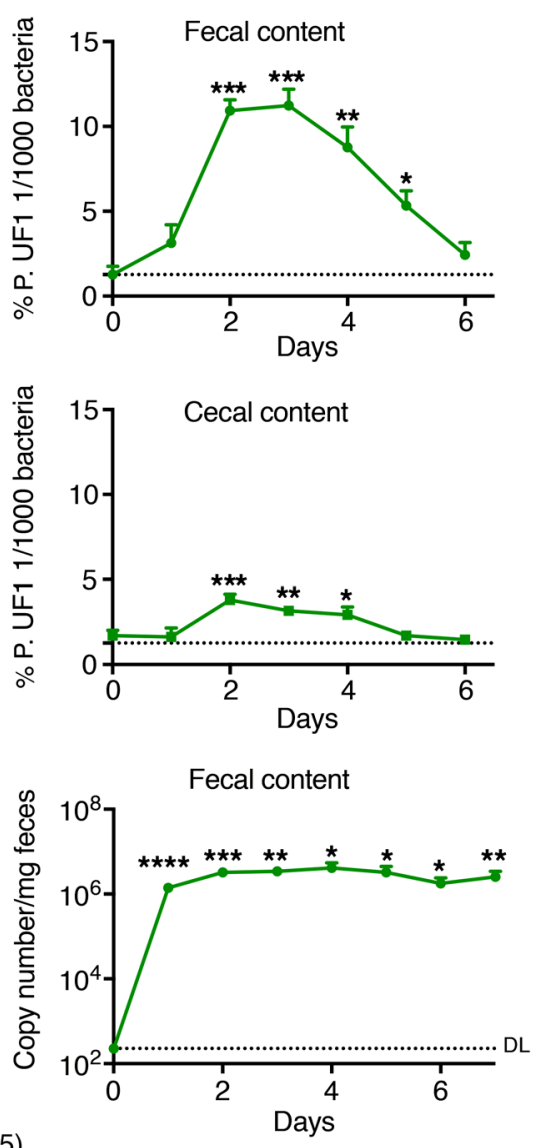

Figure 2. Characterization of P. UF1 bacterium. (A) Genome sequence comparison of isolated P. UF1 with known $P$. freudenreichii spp. Shermanii CIRM-BIA1 (red) and P. freudenreichii spp. freudenreichii DSM20271 ${ }^{\top}$ (blue). Prophage was identified by PHAST (green). Sequence identity between $P$. freudenreichii strains and P. UF1 is shown. (B) Correlation of the annotated genome sequence on the RAST platform with different metabolic pathways of P. UF1. Numbers in parentheses indicate numbers of annotated genes belonging to subcategories of pathways. (C and $\mathbf{D})$ C57BL/ 6 mice $(n=3)$ were gavaged with P. UF1 ( $10^{9}$ CFU/mouse) 1 time, and mice were sacrificed every day to detect P. UF1 in the fecal (C) and cecal (D) contents. (E) GF mice $(n=5)$ were gavaged with P. UF1 ( $10^{9} \mathrm{CFU} /$ mouse) 1 time, and fecal samples were collected every day to detect P. UF1. DL indicates the qPCR detection limit. Asterisks indicate statistical significance compared with day 0 . Data are representative of 1 (E) or 3 (C and $\mathbf{D})$ independent experiments. Error bars indicate mean \pm SEM. ${ }^{*} P<0.05 ;{ }^{* *} P<0.01 ;{ }^{* *} P<0.001 ;{ }^{* * *} P<0.0001$, 2-tailed unpaired $t$ test (C-E).

P. UF1 (2.63 Mb) encodes for critical enzymes involved in fermentation, catabolism, and biosynthetic pathways for all amino acids (Figure 2B). Furthermore, introducing this bacterium to conventional C57BL/6 mice via a single oral gavage resulted in transient gut colonization, potentially due to bacterial competition for space and nutritional resources $(15,16)$, whereby this bacterium was no longer detectable in the fecal or cecal contents of C57BL/6 mice after 5 to 6 days (Figure 2, C and D). In contrast, P. UF1 permanently colonized the gut of germfree (GF) mice, as detected in the fecal contents of these mice until day 7 (Figure 2E). Such colonization in GF mice was expected, as P. UF1, like other beneficial (e.g., Bifidobacterium bifidum) (17) or pathogenic bacteria (e.g., Citrobacter rodentium) (18), demonstrates the same trend due to the lack of competitive commensal bacteria.

Regulation of intestinal proinflammation by $P$. UF1. To explore the potential immunologic differences caused by the microbiota of the aforementioned cohorts, fecal microbes derived from HBMF and FF preterm infants were transfaunated into GF mice
(19). GF mice receiving HBMF preterm infants' microbiota did not exhibit the enhanced proinflammation (e.g., IL-1 $\beta$ ) in colonic DCs that was observed in the GF mice transfaunated with FF preterm infants' microbiota (Supplemental Figure 1A; supplemental material available online with this article; https://oi.org/10.1172/ JCI95376DS1). Moreover, transfaunating GF mice with HBMF preterm infants' microbiota increased Th17 cells and sustained IL-10+ Tregs, a trend that was less evident in GF mice transfaunated with FF preterm infants' microbiota (Figure $3 \mathrm{~A}$ ). Not only were proinflammatory DC responses attenuated upon P. UF1 gavage of GF mice transfaunated with FF preterm infants' microbiota, but Th17 cells and IL-10 ${ }^{+}$Tregs were also significantly increased to the levels seen in GF mice transfaunated with HBMF preterm infants' microbiota (Supplemental Figure 1A and Figure 3A). Additionally, the composition of the gut microbiota in GF mice transfaunated with FF preterm infants' microbiota and subsequently gavaged with P. UF1 showed changes in bacterial organization, including decreased levels of $\gamma$-Proteobacteria, when compared 
A
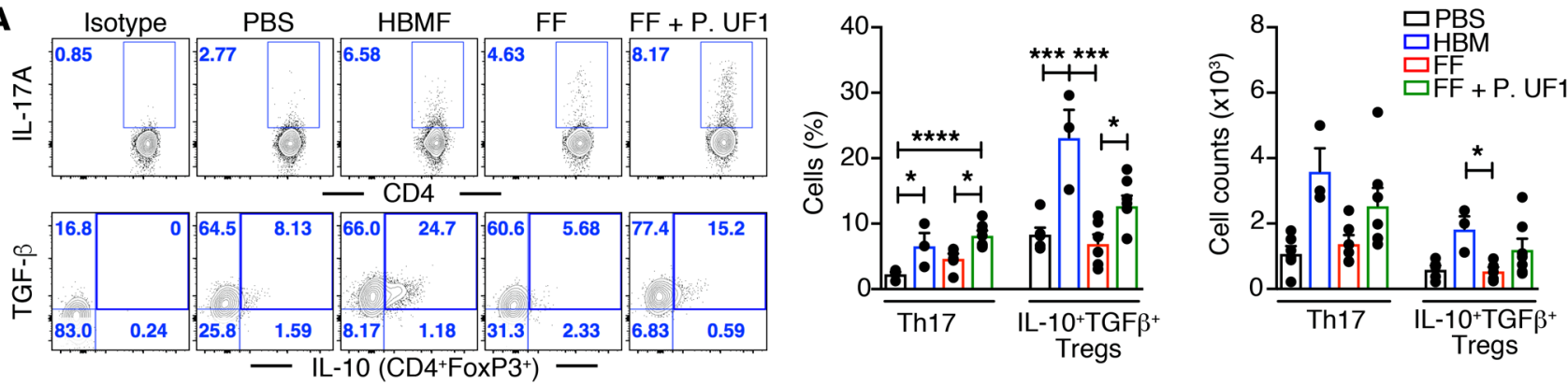

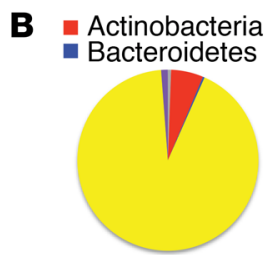

HBMF

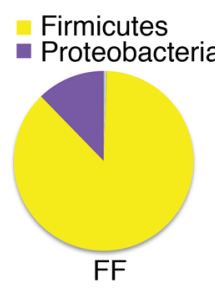

Unassigned
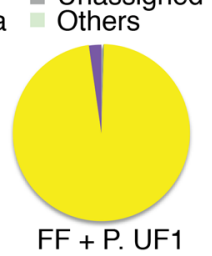

C Unclassified Enterococcaceae Bifidobacterium Bifidobacteriales Enterococcaceae nterococcaceae Enterococcus Proteobacteria Gammaproteobacteria Enterobacteriaceae Unclassfied Enterobacteriaceae Unclassified Člostridiales
Unclassified Clostridiales

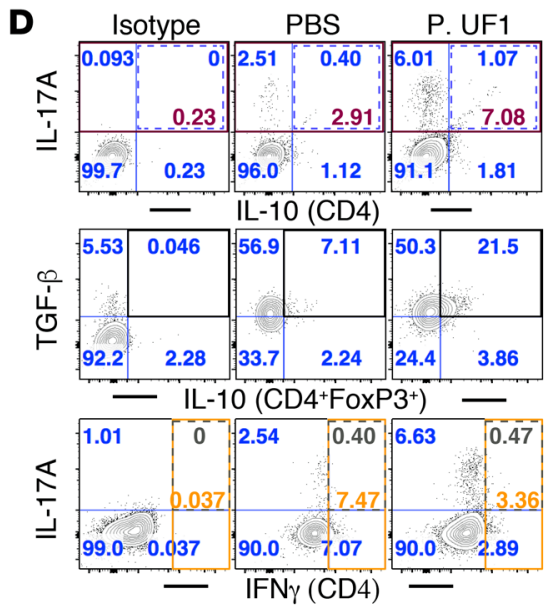

E

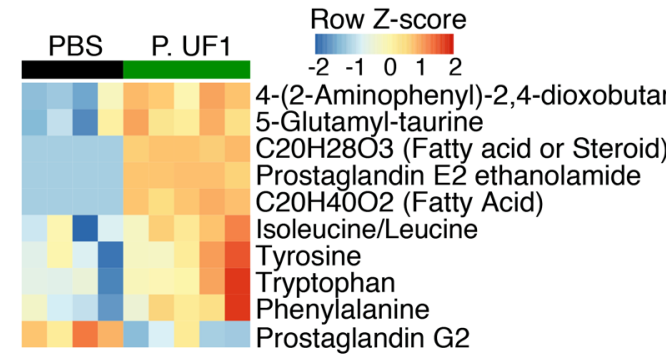

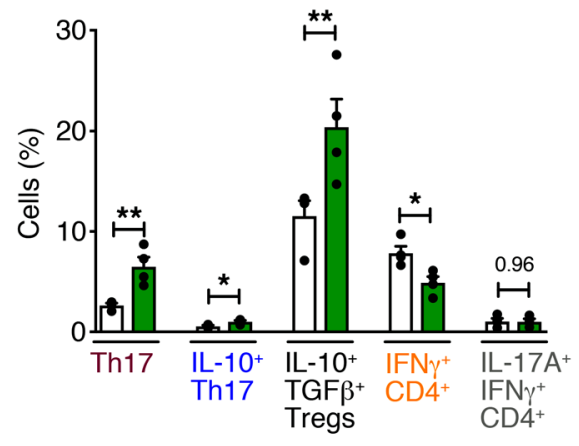

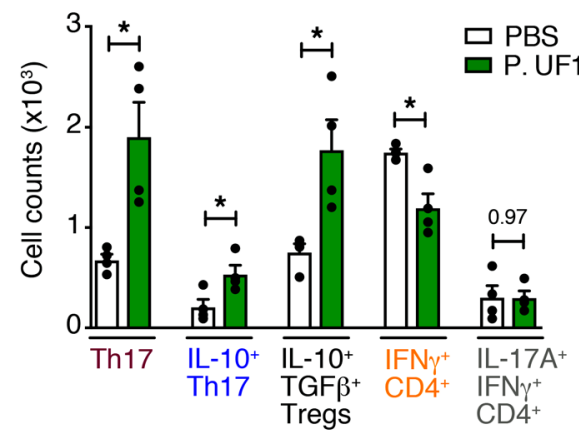

F Aspartate and asparagine Starch and Sucrose Sialic acid Porphyrin Galactose Tryptophan Vitamin B9 (folate) Butanoate Vitamin B6 (pyridoxine) C21-steroid hormone

Metabolisms

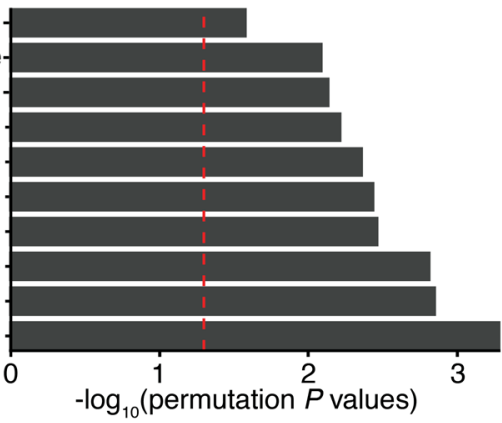

Figure 3. Modulation of colonic immune responses by HBMF preterm infants' microbiota. (A-C) GF mice were transfaunated with HBMF preterm infants' microbiota (blue), FF preterm infants' microbiota (red), or FF preterm infants' microbiota plus 4 treatments with P. UF1 (green) or were left untransfaunated (black). Colonic immune responses and microbiota were analyzed 2 weeks later. Representative data of flow plots, percentages, and total cell counts of Th17 cells and IL-10+TCF- $\beta^{+}$Tregs (A). Microbial phyla structure (B) and LDA analysis (C) of fecal samples from indicated group. (D) GF mice were monoassociated with P. UF1 (green) or gavaged with PBS (white), and induced colonic immune responses were analyzed. Representative data of flow plots, percentages, and total cell counts of Th17 cells, IL-10+TCF- $\beta^{+}$Tregs, and IFN- $\gamma^{+}$CD4 $4^{+}$cells (Th1 cells). (E and F) Heatmap of selected metabolites differentiating fecal samples from PBS- and P. UF1-gavaged GF mice (E), and significant metabolic pathways of fecal samples from PBS- versus P. UF1-gavaged GF mice (F). Red dashed line shows permutation of $P=0.05$. Data are pooled from 2 independent experiments ( $n=3-7$ mice/group, A-C) or representative of 3 ( $n=4-5$ mice/group, $\mathbf{E}$ and $\mathbf{F})$ or 6 ( $n=4$ mice/group, D) independent experiments. Error bars indicate mean $\pm \mathrm{SEM}$. ${ }^{*} P<0.05 ;{ }^{* *} P<0.01 ;{ }^{* * *} P<0.001 ;{ }^{* * *} P<0.0001, \mathrm{ANOVA}$ plus Tukey's post test (A) or 2-tailed unpaired $t$ test (D).

with the FF preterm infants' microbiota, indicating the potential influence of P. UF1 on the FF preterm infants' microbiota (Figure 3, B and C). The same trend was also observed in GF mice that were transfaunated with HBMF preterm infants' microbiota, wherein $\gamma$-Proteobacteria levels were also decreased, possibly by the elevated growth of Actinobacteria, since beneficial bacteria may compete with indigenous pathobionts (e.g., $\gamma$-Proteobacteria) $(20-22)$ in the given environment of GF mice (Figure 3B). To 
A

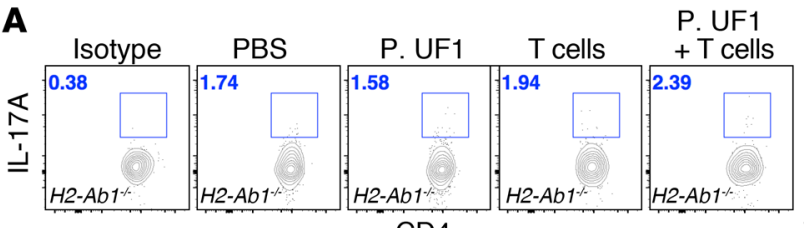

CD4

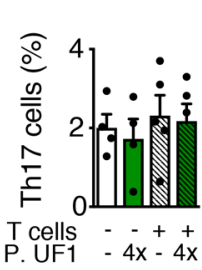

B

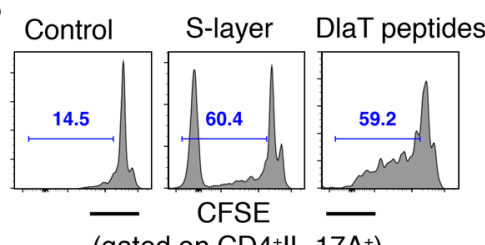

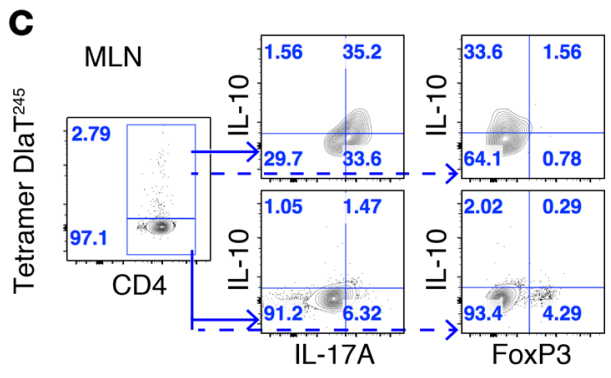
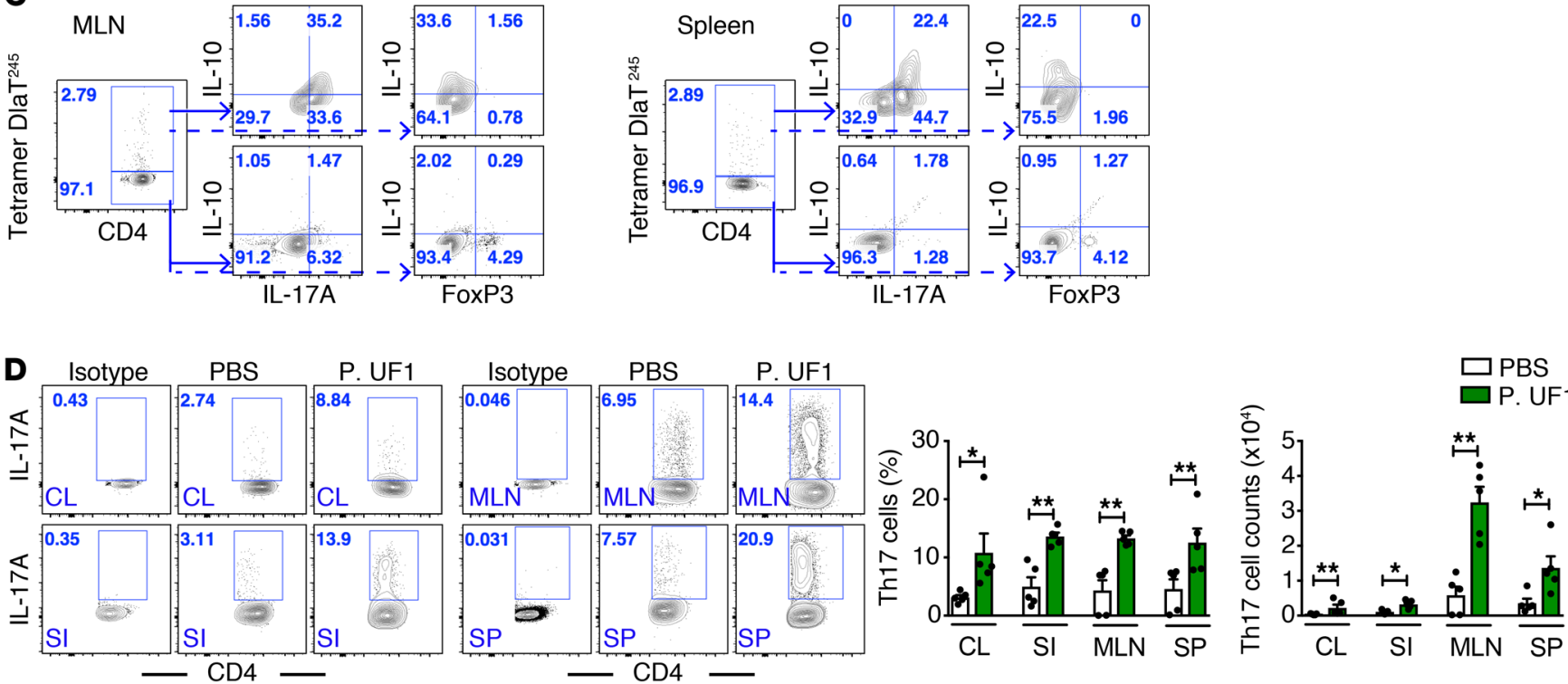

Figure 4. Induction of Th17 cell differentiation by P. UF1. (A) CD4 ${ }^{+}$splenic cells derived from C57BL/6 mice gavaged with P. UF1 were transferred into

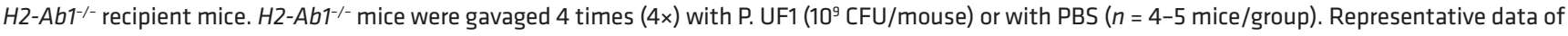
flow plots, percentages, and total cell counts of colonic Th17 cells. (B) Splenic CD4+ T cells were sorted and labeled with CFSE. T cells were cocultured with pulsed BMDCs with S-layer $(10 \mu \mathrm{g} / \mathrm{ml})$ or DlaT peptides $(20 \mu \mathrm{g} / \mathrm{mg})$ for 5 days. Cells were stained and analyzed by flow cytometry. Representative histogram plots of CFSE-labeled IL-17A+ CD4 ${ }^{+}$T cells. (C) C57BL/6 mice were gavaged 4 times with P. UF1. Splenic and MLN tetramer DlaT ${ }^{245+}$ cells were enriched with phycoerythrin (PE) beads and analyzed. Representative plots depicting the percentage of DlaT $\mathrm{T}^{245+}$ tetramer CD4+ $\mathrm{T}$ cells with a majority of IL-17A ${ }^{+} \mathrm{IL}-10^{+} \mathrm{T}$ cells, but negative for FoxP3 ${ }^{+} T$ cells. (D) Th17 cell differentiation from naive splenic Thy1.1+CD4+CD45RB hi cells in Thy1.2 ${ }^{+}$Rag $1^{-1-}$ mice gavaged with P. UF1 or PBS. Th17 cell differentiation was analyzed in the colon (CL), small intestine (SI), MLN, and spleen (SP) ( $n=5$ mice/group). Data are representative of 2 (D) or 3 $(\mathbf{A}-\mathbf{C})$ independent experiments. Error bars indicate mean \pm SEM. ${ }^{*} P<0.05 ;{ }^{* *} P<0.01$, ANOVA plus Tukey's post test (A) or Mann-Whitney $U$ test (D).

specifically investigate P. UF1-induced Th17 cells, GF mice were monoassociated with P. UF1 (4 oral gavages with $10^{9} \mathrm{CFU} /$ mouse, every 3 days), and DC and T cell responses were analyzed. Once again, proinflammatory cytokines (e.g., IL-1 $\beta$ ) promoting intestinal pathology (23) were decreased in colonic DCs (Supplemental Figure 1B and Supplemental Figure 11). Additionally, cytokine transcripts (e.g., IL-1 $\beta$ ) were not only diminished in colonic tissues of GF mice monoassociated with P. UF1, but also in sorted colonic DCs (Supplemental Figure 2, B and C). The generation of Th17 cells, particularly IL-10+IFN- $\gamma^{-}$Th17 cells, and IL- $10^{+}$Tregs was also substantially increased in GF mice monoassociated with P. UF1, while the IFN- $\gamma^{+} \mathrm{CD} 4^{+} \mathrm{T}$ cell response (Th1 cells) was decreased compared with that in the control groups (Figure 3D and Supplemental Figure 12), suggesting that P. UF1 not only critically regulates DCs, but also specifically augments the formation of Th17 cells. Such differential dependency of the Th17 cells on P. UF1 was then highlighted in GF mice monoassociated with P. UF1 or E. coli JM109 (24). Here, E. coli JM109, when compared with P. UF1, did not induce Th17 cell differentiation (Supplemental Figure 1C). Furthermore, tryptophan, tyrosine, and phenylala- nine were increased in the fecal samples of the GF mice monoassociated with P. UF1 (Figure 3E), potentially regulating Th17 cell homeostasis $(25,26)$. Accordingly, pathway analyses $(27)$ revealed that these enriched gut metabolites (Figure 3F) are highly involved in C21-steroid hormone biosynthesis (28), tryptophan metabolism, multiple vitamin biosynthetic pathways (e.g., vitamin $\mathrm{B}_{12}$ ) (29), and porphyrin biosynthesis (30).

Bacterial dihydrolipoamide acetyltransferase drives Th17 cells. Sensing receptor interactions with the bacterial surface layer (S-layer) stimulate intestinal DCs, leading to $\mathrm{T}$ cell activation (31-34). Activated $\mathrm{T}$ cells, in general, and antigen-specific Th17 cells, in particular, are tightly controlled by gut commensals, including segmented filamentous bacteria (SFB) $(35,36)$ along with regulated cytokines (e.g., IL-6, TGF- $\beta$, IL-1 $\beta$ ) (37). To elucidate the role of the P. UF1 $\mathrm{S}$-layer in Th17 cell differentiation, the S-layer was isolated by guanidine hydrochloride ( $\mathrm{GdnHCl})$ and characterized by mass spectrometry. Dihydrolipoamide acetyltransferase (DlaT) was found to be one of the major S-layer proteins, from which three 15-mer peptides were deduced exhibiting high affinity for MHC II. Interestingly, DlaT is critically involved in pyruvate decarboxylation, which links glycoly- 
A

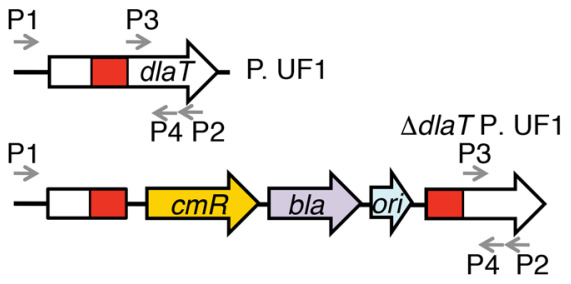

$\mathbf{E}$

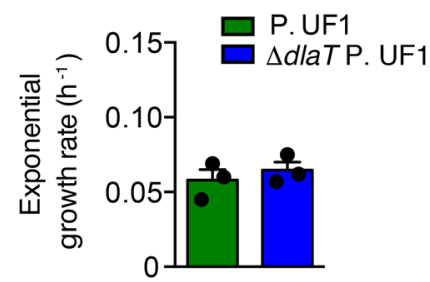

B

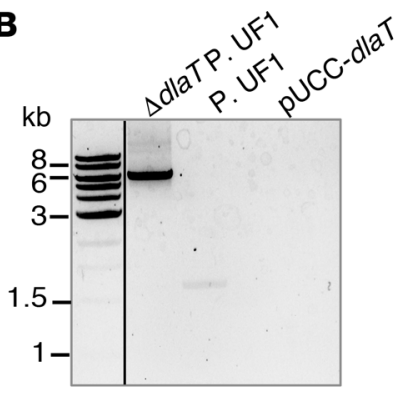

$\mathbf{F}$

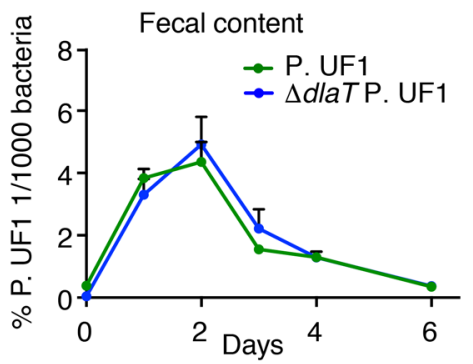

C

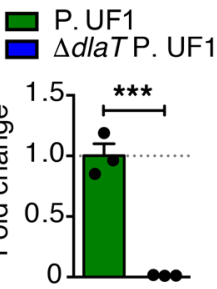

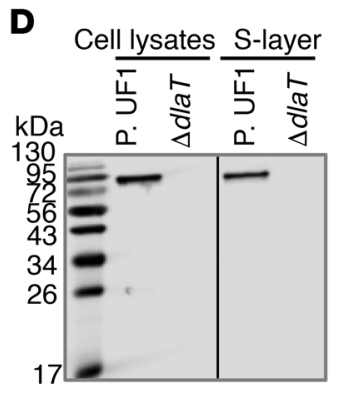

G
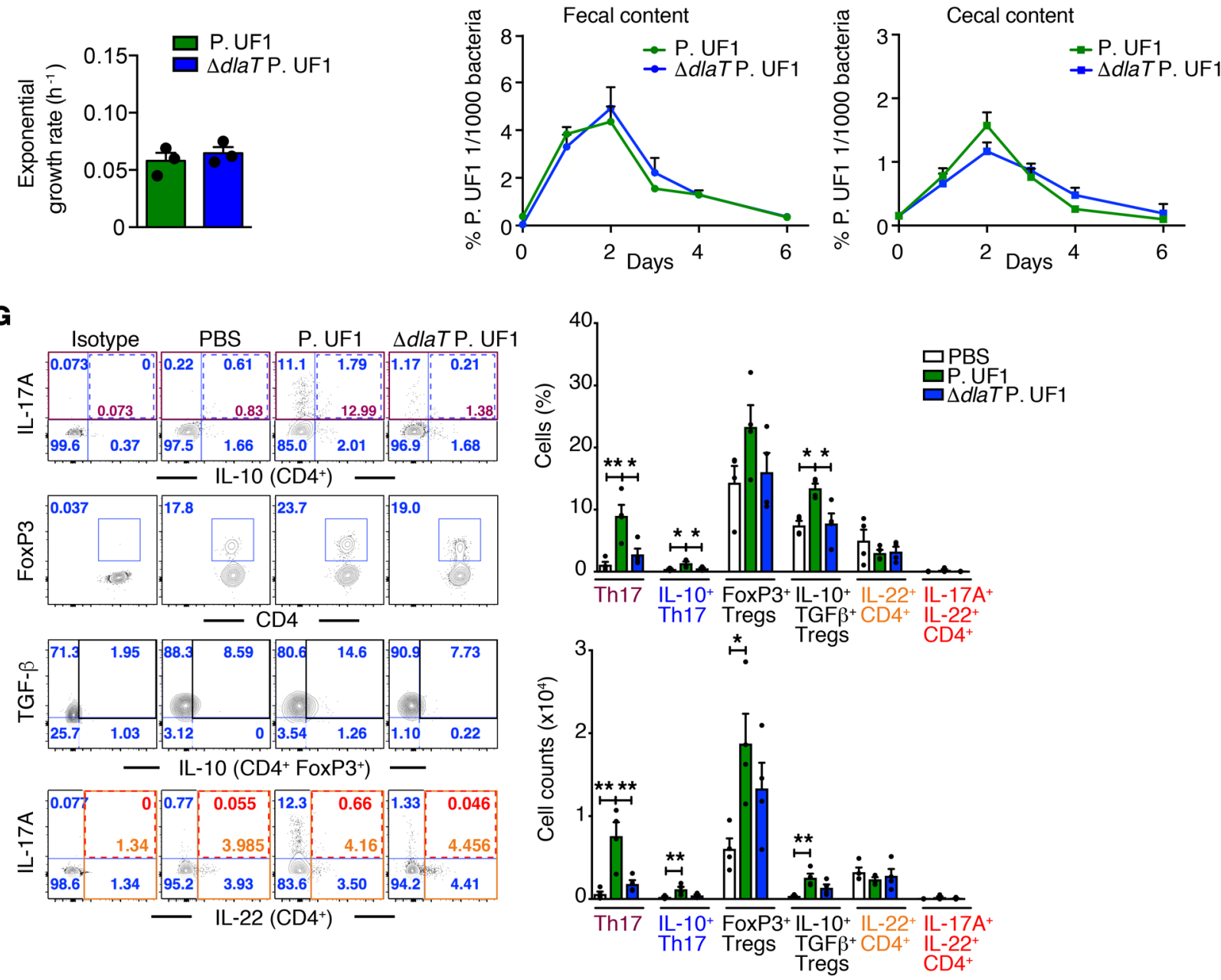

Figure 5. Differentiation of DlaT-specific Th17 cells. (A) Genetic organization of the dlaT locus from P. UF1 and $\Delta$ dlat P. UF1. P1, P2, P3, and P4 primers was used for identifying $\triangle$ dlaT P. UF1. cmR, chloramphenicol resistance gene (P. UF1); bla, ampicillin resistance gene (E. coli); ori, replication origin of pUC19 plasmid. (B) PCR amplification of dlaT with primers P1/P2. (C) Quantitative reverse-transcriptase PCR (qRT-PCR) analyses of dlaT mRNA expression using primers P3/P4 ( $n=3$ samples/group). (D) Western blot analysis of DlaT expression in the cell lysates and S-layer using polyclonal anti-DlaT antibodies. (E) Exponential growth rate of P. UF1 and $\Delta d l a T$ P. UF1 in MRS-lactate medium ( $n=3$ samples/group). (F) Colonization of P. UF1 and $\Delta d l a T$ P. UF1 in C57BL/6 mice ( $n=2$ mice/group). (G) GF mice were gavaged with P. UF1 (green), $\triangle$ dlaT P. UF1 (blue), or PBS (white). Colonic cell immune responses were analyzed 2 weeks later ( $n=4$ mice/group). Representative data of flow plots, percentages, and total cell counts of Th17 cells, CD4 ${ }^{+}$FoxP3 ${ }^{+}$Tregs, IL-10+TCF- $\beta^{+}$Tregs, and IL-22+CD4+ $T$ cells. Data are representative of 1 (F) or 3 (B-E and $\mathbf{G})$ independent experiments. Error bars indicate mean $\pm \mathrm{SEM} .{ }^{*} P<0.05 ;{ }^{*} P<0.01$; ${ }^{* * *} P<0.001,2$-tailed unpaired $t$ test ( $\mathbf{C}$ and $\mathbf{E}$ ) or ANOVA plus Tukey's post test (G).

sis to the citric acid cycle (38); however, this protein could also be expressed by other, yet to be identified mechanism(s) on the S-layer to exert immune activation. Importantly, we demonstrate the dependency of P. UF1 S-layer-induced Th17 cells in $\mathrm{H}_{2}-\mathrm{Ab1}^{+/ /}$sufficient mice $\left(\mathrm{MHC} \mathrm{II}^{+/+}\right)$(Supplemental Figure 3A). However, this was not observed in $\mathrm{H} 2-\mathrm{Ab} 1^{-/}$deficient mice gavaged with P. UF1 alone or with P. UF1 plus adaptively transferred $\mathrm{CD} 4^{+} \mathrm{T}$ cells (Figure $4 \mathrm{~A}$ ). Thus, these data once again illuminate the critical role of MHC II in antigen-specific $\mathrm{CD} 4^{+} \mathrm{T}$ cell differentiation, as also previously demonstrated for SFB-dependent Th17 cells (39). 
A

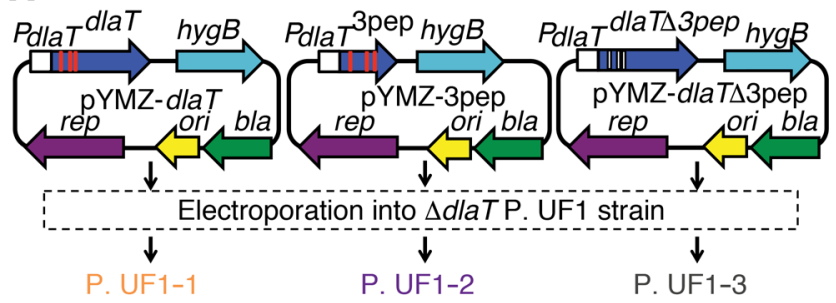

B

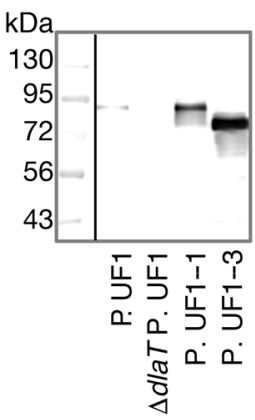

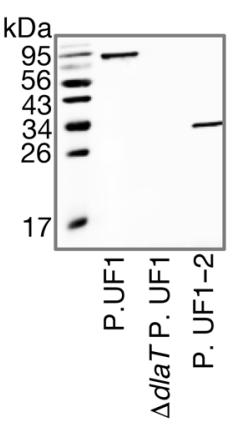

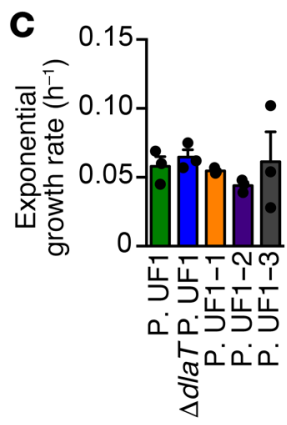

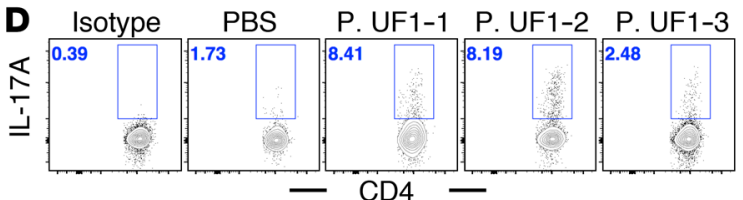
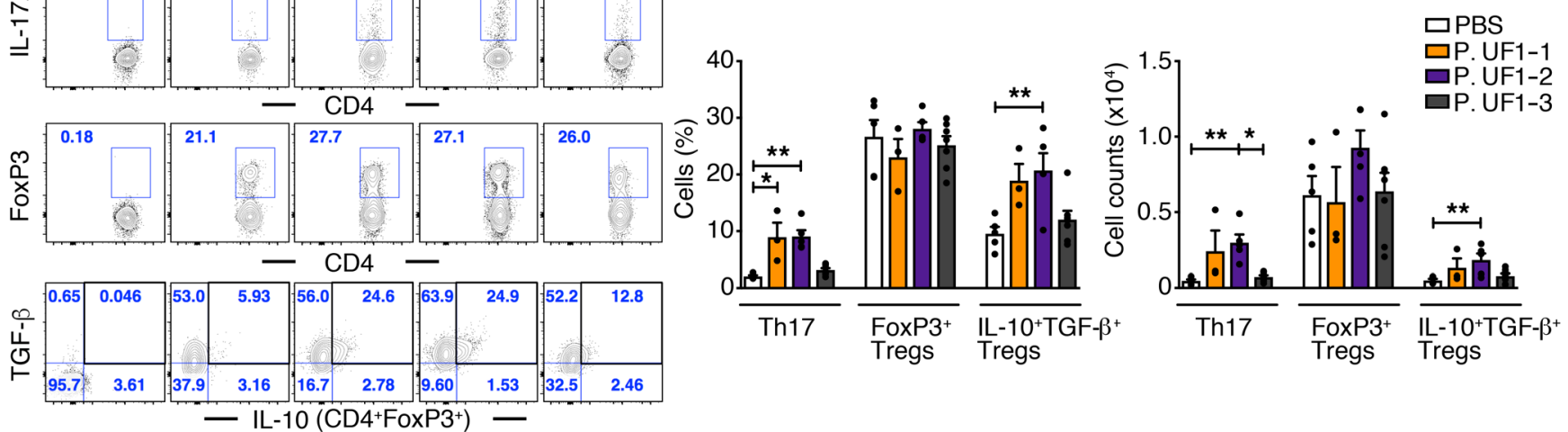

$\mathbf{F}$
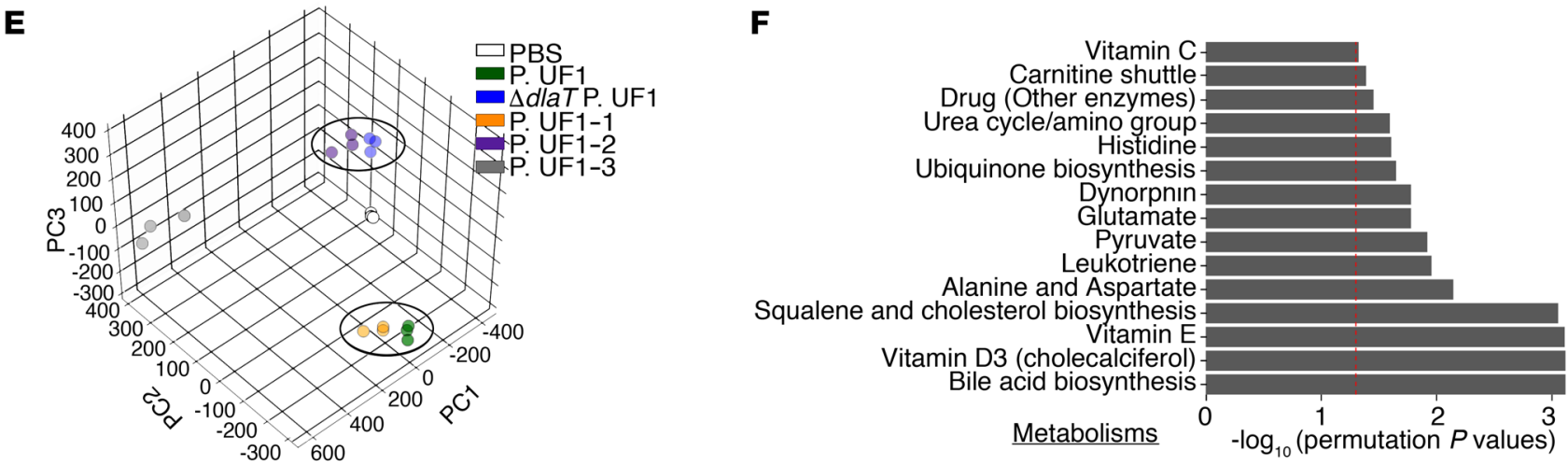

Figure 6. Restoring Th17 cell differentiation by complementation of $\triangle d$ llaT P. UF1 with dlaT. (A) Complementation of $\triangle$ dlaT P. UF1 with the dlaT gene (P. UF1-1, orange), the 3 dlaT peptides (P. UF1-2, purple), and the dlaT gene minus the 3 peptides (P. UF1-3, gray). (B) Western blot analysis of DlaT expression and the 3 peptides in cell lysates of the complemented strains using polyclonal anti-DlaT antibodies. (C) Exponential growth rate of the complemented strains in MRSlactate medium ( $n=3$ samples/group). (D) GF mice were gavaged with P. UF1-1, P. UF1-2, P. UF1-3 (109 CFU/mouse), or PBS on days 0, 3, 6, and 9. Two weeks later, induced colonic immune responses were analyzed. Representative data of flow plots, percentages, and total cell counts of Th17 cells, CD4 ${ }^{+}$FoxP3 ${ }^{+}$Tregs, and IL-10+TCF- $\beta^{+}$Tregs. (E and F) Fecal sample metabolomic analyses of GF mice gavaged with aforementioned complemented strains or PBS. PCA of fecal sample metabolites (E) and comparison of significant pathways between P. UF1: P. UF1-1 and $\triangle$ dlaT P. UF1: P. UF1-2 (F). Red dashed line shows permutation $P$ value of 0.05. Data are pooled from 2 independent experiments $(n=3-7$ mice/group, $\mathbf{D})$ or are representative of 1 ( $n=3$ mice/group, $\mathbf{E}$ and $\mathbf{F})$ or 3 (B and $\mathbf{C}$ ) independent experiments. Error bars indicate mean \pm SEM. ${ }^{*} P<0.05$; ${ }^{* *} P<0.01$, ANOVA plus Tukey's post test (C) or Kruskal-Wallis plus Dunn's post test (D).

Additionally, it has recently been reported that bacterialinduced metabolites support the differentiation of T cells, including Th17 cells (40). To demonstrate whether P. UF1-induced metabolites can prime Th17 cell differentiation, metabolomic analyses of P. UF1-gavaged $H 2-A b 1^{-/}$mice compared with $H 2-A b 1^{-/}$mice plus $\mathrm{CD}^{+}{ }^{+} \mathrm{T}$ cells and PBS-gavaged $\mathrm{H2}-\mathrm{Ab} \mathrm{1}^{-/}$mice were performed. Data revealed different metabolic pathways, particularly in tryptophan and butanoate metabolism; however, no Th17 cells were promoted in the $\mathrm{H2}-\mathrm{AbI}^{-/-}$mice lacking MHC II (Supplemental Figure 3, B and C), once again highlighting the critical role of MHC II in differential T cell activation (41). Furthermore, cytokines (e.g.,
IL-1 $\beta$, IL-6) alone were not sufficient to induce Th17 cells in mice that were gavaged with the S-layer P. UF1, denoting that the S-layer and its deduced peptides are essential in Th17 cell differentiation (Supplemental Figure 3A).

To further evaluate S-layer-specific Th17 cells, C57BL/6 mice were gavaged with P. UF1. Subsequently, splenic CD4 ${ }^{+}$ $\mathrm{T}$ cells were sorted and cocultured with bone marrow-derived DCs pulsed with the S-layer or with the 3 DlaT-peptides. Data demonstrate the differential DlaT positivity for only splenic and mesenteric lymph node (MLN) Th17 cells, but not IL-10+ Tregs, using the DlaT-specific tetramer ${ }^{245}$ (Figure 4, B and C). To specifi- 
A P. UF1+ $\triangle$ dlaTP. UF1+

Isotype $\Delta$ actA L.m $\Delta$ actA L.m $\quad$ actA L.m $\Delta$ actA L.m $m^{3 p e p}$
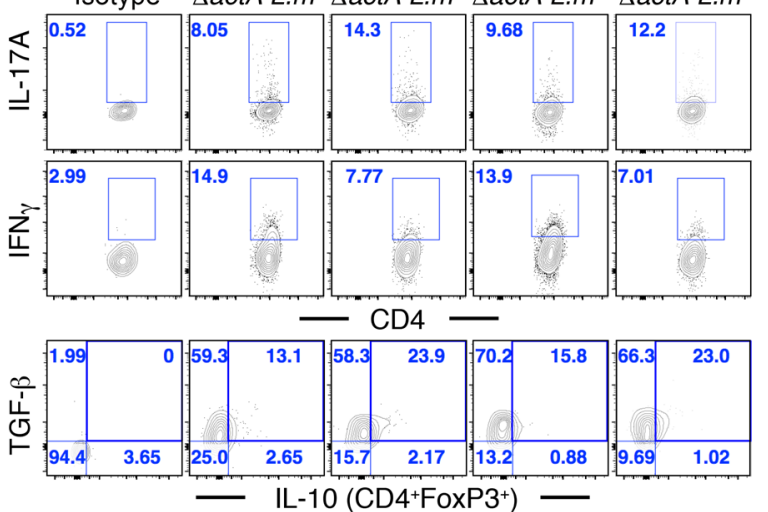

B

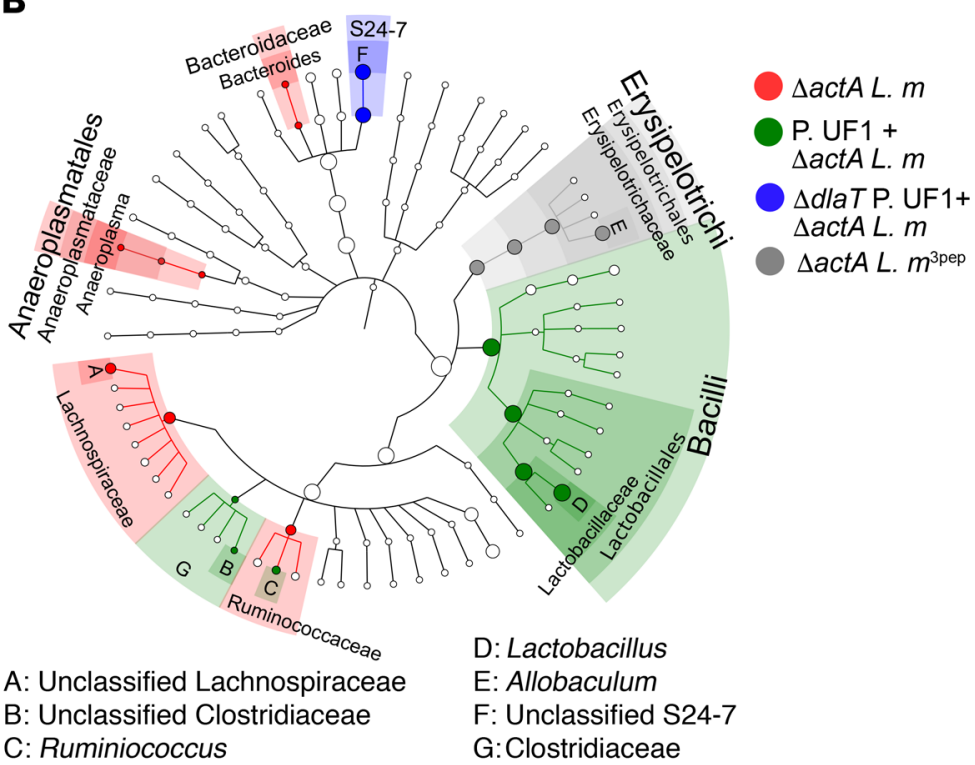

Foxp3 ${ }^{\text {DTR }}$ mice with no DT injection

$\triangle a c t A$ L.m $\triangle a c t A L \cdot m+\mathrm{P}$ UF 1 $\Delta$ actA L. $m+\Delta$ dlaTP. UF1 $\Delta$ actA L. $m^{3 \mathrm{pep}}$
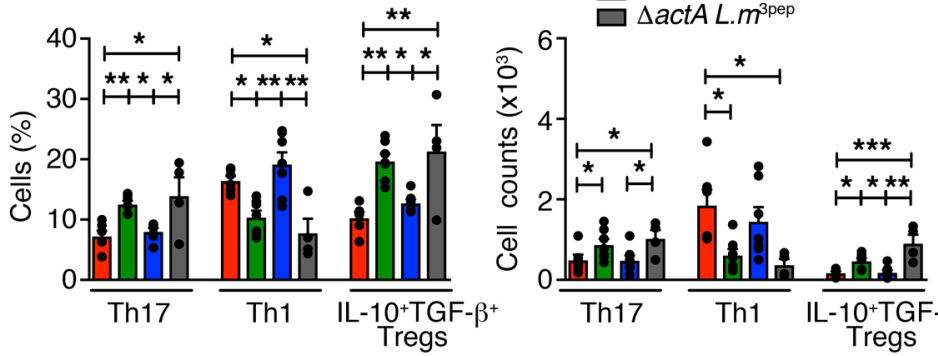

Th17

Th1

IL-10+TGF- $\beta$

C

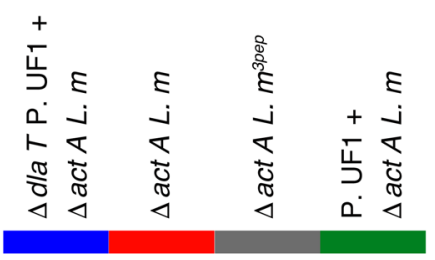

Row Z-score

$\begin{array}{lllll}-2 & -1 & 0 & 1 & 2\end{array}$

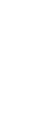

Tyrosine

Phenylalanine

Tryptophan

Citrulline

Methionine

Lysine

Arginine

Betaine/Valine

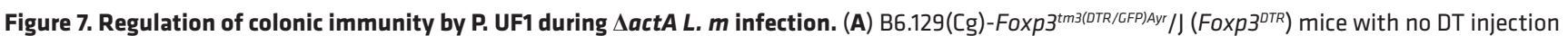
were gavaged with PBS (red), P. UF1 (green), or $\triangle$ dlaT P. UF1 (blue) and orally infected with $\triangle a c t A ~ L$. $m$. One group of mice was orally infected with $\triangle a c t A$ L. $m^{\text {3pep }}$ (gray). Representative data of flow plots, percentages, and total counts of colonic Th17 cells, Th1 cells, and FoxP3+IL-10+TCF- $\beta^{+}$Tregs. (B) Taxonomic cladogram of fecal microbiota from experimental groups $(P<0.05$, Kruskal-Wallis test) from groups of C57BL/6 mice gavaged with P. UF1 or dlaT P. UF1 and orally infected with $\triangle a c t A$ L. $m$ as described in A. (C) Heatmap of selected fecal metabolites $(P<0.05,1$-way ANOVA) from mouse groups as described in A. Data are pooled from 2 independent experiments ( $n=4-7$ mice/group, A) or representative of 2 independent experiments ( $n=3$ mice/group, B and $\mathbf{C}$ ). Error bars indicate mean \pm SEM. $P<0.05 ;{ }^{*} P<0.05$; ${ }^{*} P<0.01$; ${ }^{* * *} P<0.001$, ANOVA plus Tukey's post test (A).

cally determine the differentiation of Th17 cells by P. UF1, sorted naive splenic Thy $1.1^{+} \mathrm{CD} 4{ }^{+} \mathrm{CD} 45 \mathrm{RB}^{\text {hi }}$ cells were transferred into P. UF1- or PBS-gavaged Thy1.2 $2^{+}$Rag1 $1^{-/-}$mice (42) lacking mature $\mathrm{T}$ and $\mathrm{B}$ cells (43). Data document the differentiation of Th17 cells from Thy $1.1^{+} \mathrm{CD} 4^{+} \mathrm{CD} 45 \mathrm{RB}^{\text {hi }} \mathrm{T}$ cells by P. UF1 in the intestines, MLNs, and spleens of Thy1.2 $2^{+} \operatorname{Rag1}^{-/-}$mice (Figure 4D).

To investigate the mechanistic paradigm associated with DlaT-specific Th17 cell differentiation, the dlaT gene was deleted from the bacterial chromosome by homologous recombination with a single crossover event (44), resulting in the $\Delta$ dlaT P. UF1 (Figure 5, A-D). Importantly, the bacterial growth kinetics and detection of $\triangle d l a T$ P. UF1 in conventional C57BL/6 mouse fecal and cecal contents were comparable to P. UF1 (Figure 5, E and F). When compared with P. UF1, GF mice monoassociated with $\Delta d l a T$ P. UF1 exhibited significantly increased expression of IL-1 $\beta$,
IL-6, and IL-12/23p40 in colonic DCs and colonic tissue (Supplemental Figure 2, A-C). $\Delta d l a T$ P. UF1 also substantially abrogated Th17 cells, while IL-10+ Tregs were generally comparable to those of PBS-gavaged mice, suggesting a crucial role for DlaT in IL-10 ${ }^{+}$ Th17 cell differentiation. Such P. UF1-induced Th17 cells did not significantly express IL-22 (45) (Figure 5G).

To delineate any potential changes in $\Delta d l a T$ P. UF1 transcriptomic machinery that may affect gut homeostasis, the transcriptic and metabolic changes in $\triangle d l a T$ P. UF1 compared with P. UF1 were analyzed. Deletion of dlaT in P. UF1 resulted in significant transcriptic changes involved in the glycolytic and multiple metabolic pathways, including the metabolism of carbohydrates, nitrogen, folate, and cysteine biosynthesis (Supplemental Figure 4, A-C). More importantly, multiple tryptophan-derived metabolites (e.g., hydroxykynurenamine, formyl-acetyl-5-methoxykynurenamine) 
were significantly observed in P. UF1, but not in $\triangle d l a T$ P. UF1 (Supplemental Figure 4, D and E), potentially qualitatively influencing the regulation of $\mathrm{DC}$ and $\mathrm{T}$ cell responses.

To specifically elucidate the relevance of DlaT in Th17 cell differentiation and to exclude possible ectopic effects resulting from dlaT deletion in P. UF1, we then complemented the dlaT gene (P. UF1-1), the 3 DlaT peptide sequences (P. UF1-2), or the dlaT gene without these 3 peptides (P. UF1-3) in the $\Delta$ dlaT P. UF1 (Figure $6, \mathrm{~A}$ and $\mathrm{B}$ ), whereby the bacterial growth kinetics of these P. UF1 recombinants were comparable to those of P. UF1 (Figure 6C). GF mice were then monoassociated with P. UF1-1, P. UF1-2, or P. UF1-3. Data demonstrate the significance of DlaT and the 3 DlaT peptides, such that mice receiving P. UF1-1 or P. UF1-2, but not P. UF1-3, exhibited differentiation of Th17 cells and induction of IL-10+ ${ }^{+}$Tregs (Figure 6D). Furthermore, metabolomic analyses of P. UF1-1 and P. UF1 separated similarly from the other groups of GF mice gavaged with $\Delta$ dlaT P. UF1, P. UF1-2, P. UF1-3, or PBS, indicating a physiologic role for DlaT in P. UF1 molecular machinery supporting bacterial metabolic homeostasis (Figure 6, E and F).

Immune regulation by $P$. UF1 against Listeria monocytogenes infection. Knowing the importance of commensals in the instruction of Th17 cell differentiation $(46,47)$, the protective role of Th17 cells in response to intracellular pathogens, including Listeria monocytogenes (L. M), may demand consideration (48). To further evaluate the regulatory effects of P. UF1 on protective colonic DlaT-dependent Th17 cells against $L . m$ infection, $\Delta a c t A L . m$ was established. $\triangle a c t A L . m$ lacking the ActA polypeptide cannot interact with actin filaments and is, as a consequence, nonmotile to spread in the periphery (49). Subsequently, $\triangle a c t A L . m^{3 p e p}$ was generated by introducing pIMK2, which specifically harbors the $3 \mathrm{DlaT}$ peptides into $\triangle a c t A$ L. $m$ (Supplemental Figure 5, A and B). Indeed, $\Delta a c t A$ $L$. $m^{3 \text { pep }}$, like P. UF1, controlled DCs by attenuating proinflammatory signals (e.g., IL-1 $)$ (Supplemental Figure 5C) and regulating IFN $-\gamma^{+}$Th1 cells, potentially minimizing pathogenic inflammation and induced DlaT tetramer ${ }^{245+} \mathrm{IL}^{-1} \mathrm{O}^{+}$Th17 cell responses when compared with other mouse groups infected only with $\triangle a c t A L . m$ or gavaged with $\Delta d l a T$ P. UF1 and infected with $\triangle a c t A$ L. $m$ (Figure 7A and Supplemental Figure 6, A and B). These data highlight the critical involvement of DlaT in colonic Th17 cell differentiation, whereby its deletion significantly abated Th17 cell responses against $\triangle a c t A$ L. $m$ infection (Figure 7A). As expected, these DlaT tetramer ${ }^{245+} \mathrm{IL}-10^{+}$Th17 cells were completely diminished in $\triangle$ act $A$

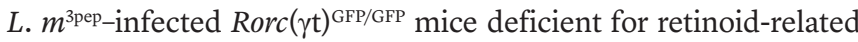
orphan receptor (ROR $\gamma \mathrm{t}$ ) (50) required for the Th17 cell lineage (Supplemental Figure 6A). Importantly, IL-10+ Tregs, which contract upon L. $m$ infection (51), were not only functionally sustained, but their frequency was markedly increased in mice orally infected with $\triangle a c t A L . m^{3 \text { pep }}$ or gavaged with P. UF1 and infected with $\triangle a c t A L . m$, potentially regulating protective CD $4^{+} \mathrm{T}$ cell subsets, including Th1 cells and Th17 cells, all of which may synergistically contribute to the resolution of this pathogenic infection (Figure 7A). Moreover, $\triangle a c t$ A L. $m$ infection was significantly reduced in mice gavaged with P. UF1 on day 4, in contrast with the other groups (Supplemental Figure 5D). When compared with that of other mouse groups, the composition of the gut microbiota of mice gavaged with P. UF1 and infected with $\triangle a c t A L . m$ demonstrates enrichment of Lactobacillus and Ruminococcus species (Figure 7B).
This may suggest that regulation of pathogen-induced inflammation by P. UF1 not only plays a role in sustaining the microbiota upon intestinal infection, but may also critically affect the pattern of induced gut metabolites, including citrulline involved in nitric oxide production and innate signaling (52), ultimately controlling protective immune responses to $\triangle a c t A L . m$ infection. Such regulatory responses were not observed in mice gavaged with $\triangle d l a T$ P. UF1 or in $\triangle a c t A$ L. $m$-infected mice (Figure 7C).

To demonstrate the role of P. UF1-induced IL- $10^{+}$Tregs in the regulation of gut homeostasis, the frequency of these cells was significantly reduced in $\mathrm{B} 6.129(\mathrm{Cg})-\mathrm{Foxp}^{\text {tm3(DTR/GFP)Ayr }} / \mathrm{J}$ mice (Foxp3 ${ }^{\text {DTR }}$ mice) through diphtheria toxin (DT) injection (53). Conversely, regulated immunity against $\triangle a c t A L . m$ infection was significantly diminished when compared with functional Tregs from mice with no DT injection (Figure 7A and Supplemental Figure 7, A and B), again highlighting the regulatory role of sustained P. UF1induced IL-10 ${ }^{+}$Tregs in controlling inflammatory responses (54) against $\triangle a c t A$ L. $m$ infection.

Regulation of $P$. UF1-induced immunity requires SIGNR1. To identify the cognate receptor that binds to the P. UF1 S-layer, governing DC function and subsequent regulation of T cells, including Th17 cells, the focus was centered on C-type lectin receptors (CLRs), particularly SIGNR1 (CD209b). SIGNR1 recognizes microbes via lipoglycan moieties inducing regulatory signals controlling DCs to initiate T cell activation (55). In fact, SIGNR1, but not SIGNR3 (CD209d) (56), was identified as the binding receptor for P. UF1 when released by CHO cells in the form of a SIGNR1hFc complex (Figure 8, A-C). Binding of P. UF1 to the SIGNR1-hFc complex was significantly reduced when SIGNR1-hFc was saturated with zymozan consisting of a protein-carbohydrate complex (Figure 8D). Additionally, gavaging mice with P. UF1 upregulated SIGNR1 in colonic tissue and in DCs (Figure 8, E and F). To assess SIGNR1 involvement in limiting pathogen-induced inflammation, Signr $1^{+/+}$and Signr1 $1^{-/-}$mice were gavaged with P. UF1, $\Delta$ dlaT P. UF1, or PBS and then orally infected with $\triangle a c t A L . m$. Data illustrate the role of SIGNR1 in assuaging $\triangle a c t A ~ L . ~ m$-induced inflammation seen in P. UF1-gavaged Signr ${ }^{+/+}$mice (Figure 8G), which was not evident in P. UF1-gavaged Signr1 ${ }^{-1-}$ mice infected with $\triangle$ actA L. $m$ (Figure $8 \mathrm{G}$ and Supplemental Figure 8). Furthermore, controlled protection by P. UF1 in Signr1 ${ }^{+/+}$mice was not observed in P. UF1-gavaged Signr1-/- mice infected with $\triangle a c t A L . m$ or $L . m^{3 \mathrm{pep}}$, as reduction of $\triangle a c t A L . m$ burden was not detected in any group of $\triangle$ actA L. $m$-infected Signr1 ${ }^{-/-}$mice (Figure 8H). Comparing metabolic pathway analyses of Signr1-/- with Signr1 ${ }^{+/+}$mice also revealed notable metabolic changes, despite the shared metabolites (e.g., fatty acid metabolism) seen in both mouse strains (Supplemental Figure 9A), while no significant gut microbiota modifications were obvious in Signr1-/- mice compared with Signr1 ${ }^{+/+}$mice (Supplemental Figure 9B). Collectively, these data support the notion that SIGNR1 regulates protective immunity by potentially activating regulatory signals, including SOCS1 against pathogens (e.g., L. m) (57), whereby its deficiency promotes pathogen persistence. Accordingly, the differential suppressors of Socs1 activation were induced in the colonic tissue of Signr1 ${ }^{+/+}$mice, but not in Signr1 ${ }^{-/-}$ mice infected with $\triangle a c t$ A L. $m$ (Figure 8I). Furthermore, critical to SIGNR1 signaling is the activation of the serine/threonine kinase Raf-1; inhibition of Raf-1 downstream signaling (e.g., MEK) 
A

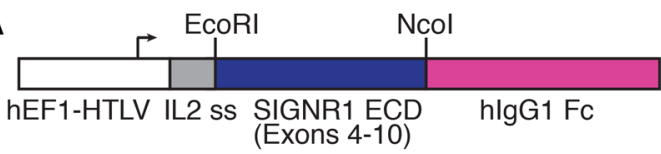

B

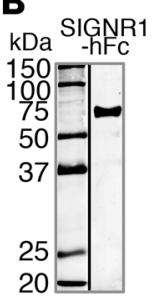

C

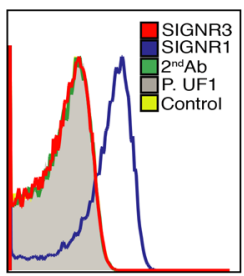

D

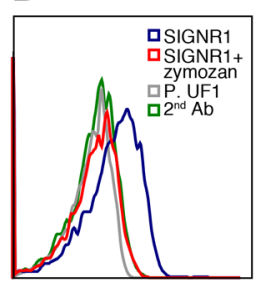

E

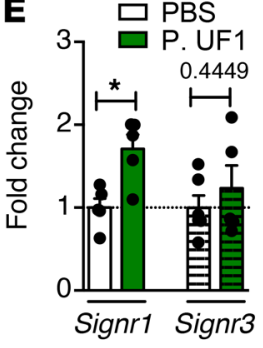

$\mathbf{F}$
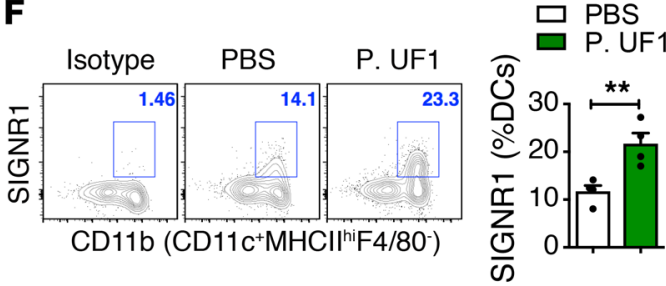

G Signr ${ }^{-1}$ mice P. UF1+ 1 dlaTP. UF1+
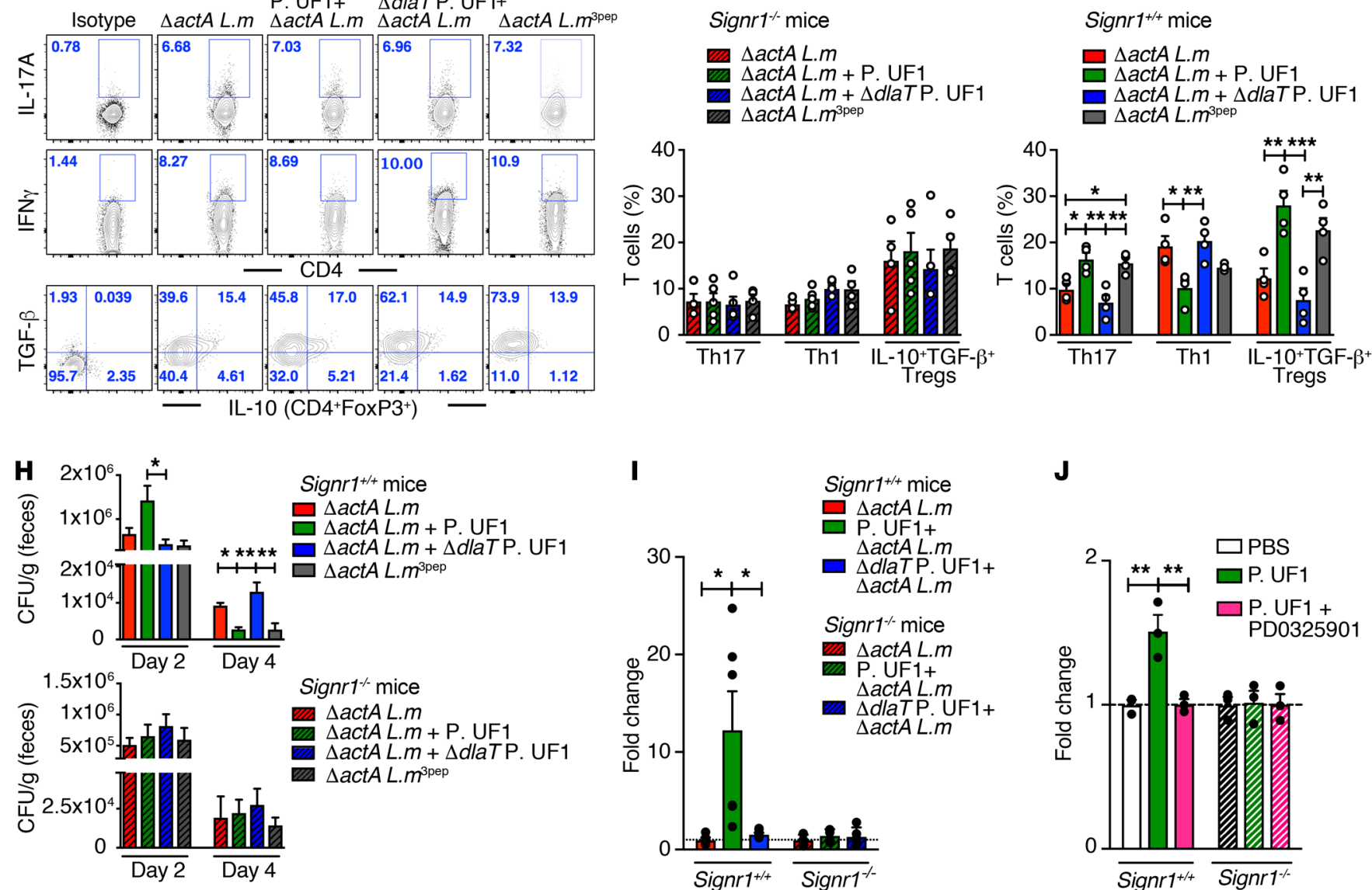

I

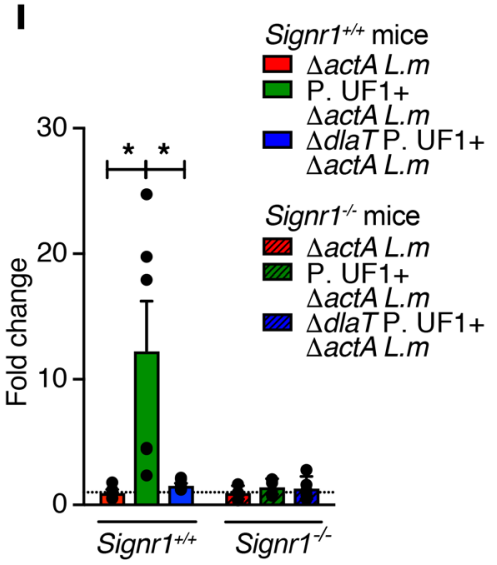

J

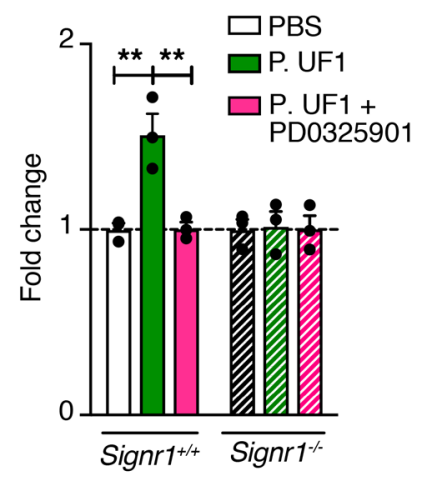

Figure 8. Regulation of intestinal immunity requires SIGNR1. (A) Depiction of SIGNR1-hFc expression. The cDNA encoding SIGNR1-extracellular domain (exons 4-10) was fused to the Fc of human IgC1. (B) Western blot analysis of SIGNR1-hFc using anti-SIGNR1 antibody. (C) Binding of P. UF1 to SICNR1 (blue), SIGNR3 (red), control fusion (yellow), or secondary (2nd) antibody (green). (D) Blocking SIGNR1-hFc binding to P. UF1 by zymozan. (E) Relative expression of Signr1 and Signr3 genes in colonic tissue from mice gavaged with P. UF1 or PBS ( $n=5$ tissues/group). (F) Representative data analyses of SICNR1 ${ }^{+}$DCs derived from mice gavaged with P. UF1 or PBS ( $n=4$ mice/group). (G) Signr ${ }^{+/+}$and Signr1 ${ }^{-/-}$mice were gavaged 4 times with PBS (red), P. UF1 (green), or $\triangle$ dlaT P. UF1 (blue) and orally infected with $\triangle a c t A L$. $m$. One group of mice was infected with actA $L$. $m^{3 p e p}$ (gray), and colonic responses were analyzed 7 days later. Representative data of flow plots, percentages, and total cell counts of Th17 cells, Th1 cells, and IL-10+TCF- $\beta^{+}$Tregs ( $n=4-5$ mice/ group). (H) Determination of $\triangle$ actA L. $m$ burden in fecal samples of indicated groups ( $n=4-5$ fecal samples/group) in Signr1+/+ and Signr1//- mice. (I) Socs1 expression by qRT-PCR in colonic tissue of $\triangle a c t A$ L. $m$-infected Signr1/++ and Signr1/- mice gavaged with P. UF1 or $\Delta d l a T$ P. UF1 ( $n=5-7$ samples/group). (J) Socs1 expression in BMDCs isolated from Signr1+/+ and Signr1-/- mice ( $n=3$ samples/group). BMDCs were treated with MEK inhibitor PD0325901 (PD) or PBS. Cells were incubated with P. UF1 (1:2 CFU) or PBS for 3 hours. Data are representative of 2 (D, E and G-J) or 3 independent experiments (B, C and F). Error bars indicate mean \pm SEM. ${ }^{*} P<0.05 ;{ }^{* *} P<0.01$; ${ }^{* *} P<0.001,2$-tailed unpaired $t$ test (E and F) or ANOVA plus Tukey post test (G-J). 
blocks SIGNR1 responses (58). To specifically delineate the signaling induced by the P. UF1 S-layer-SIGNR1 interaction controlling SOCS1 in DCs, MEK was optimally blocked by the PD0325901 inhibitor (59) within 3 hours. This led to significant reduction of Socs1 expression in P. UF1-activated DCs; in contrast, this trend was not observed in Signr1 ${ }^{-/-}$DCs (Figure 8J). These data may highlight the interplay between SIGNR1 and SOCS1 signaling to limit deleterious inflammation (57).

To evaluate any potential SIGNR1 binding to DlaT, DlaT was overproduced by P. UF1 and purified to demonstrate its interaction with SIGNR1-hFC (Supplemental Figure 9, C and D). No significant binding of DlaT to the SIGNR1-hFc complex was observed (Supplemental Figure 9E). Thus, further studies are required to elucidate the possible implication of P. UF1 S-layer mannosylation for its optimal binding to SIGNR1 to activate signals (e.g., SOCS1) in DCs controlling T cells in inflammatory conditions.

Mitigation of the intestinal inflammation by P. UF1. The functional microbiota of the mother shapes the immature immunity of the offspring during gestation (60). In contrast, alteration of the gut microbiota not only affects the immunity of the neonates, but it may also contribute to proinflammatory diseases, including NEC $(61,62)$. To determine whether P. UF1 regulates immunity in newborn mice, pregnant $\mathrm{C} 57 \mathrm{BL} / 6$ dams were gavaged with P. UF1 or PBS. Once these dams had delivered their newborn mice, all newborn mice were euthanized 5 days later to analyze immune responses. Compared with the newborn mice of PBS-gavaged dams, newborn mice of dams gavaged with P. UF1 exhibited regulated TGF- $\beta^{+} \mathrm{IL}-10^{+}$DCs (Supplemental Figure 10A) and significantly induced IL-10 ${ }^{+}$ Th17 cells and IL-10+ Tregs (Figure 9A). Additionally, to demonstrate any generation of group 3 innate lymphoid cells (ILC3) in newborn mice delivered by dams gavaged with P. UF1 or PBS, these newborns were further gavaged with P. UF1 or PBS 4 times. Twelve days later, the activation of small intestinal ILC3 of these groups of newborn mice was analyzed. Here, the frequency of IL-17A IL-22 $2^{+}$ILC3 in P. UF1-gavaged newborn mice was significantly increased when compared with control newborn mice (Supplemental Figure 10B and Supplemental Figure 13). To elucidate whether P. UF1 can mitigate pathologic conditions induced by NEC-like injury $(63,64)$, newborn mice $(5$ days of age) delivered by P. UF1-gavaged dams were compared with newborn mice of dams given PBS. The weight and survival rates were significantly increased in the newborn mice of $\mathrm{P}$. UF1-gavaged dams that were also gavaged twice with P. UF1 during NEC-like injury (Figure 9B). Newborn mice subjected to NEC-like injury also demonstrated significant villous sloughing and necrosis within the small intestine when compared with newborn mice gavaged with P. UF1 (Figure 9C). P. UF1 also significantly reduced the expression of $i N O S, I l-1 b, I l-6$, and $\mathrm{Il}-23$ transcripts implicated in NEC and tissue inflammation $(14,65)$ (Figure 9D). This correlated significantly with the regulation of IL-10 ${ }^{+}$TGF- $\beta^{+}$DCs and elevated frequencies of IL- $10^{+}$Tregs, potentially controlling Th17 cells during the height of proinflammatory NEC-like injury, a trend that was less evident in the control newborn mice subjected to NEC-like injury (Figure 9, E and F). Moreover, intestinal IL-17A+IL-22+ ILC3 activation (6668) was significantly enhanced in newborn mice gavaged with
P. UF1 compared with the control group, which could potentially account for the reduction of pathology at the mucosal surface (Supplemental Figure 10C). Further studies are required for understanding the complementary maintenance of regulatory IL17 A ${ }^{+}$IL-22 ${ }^{+}$ILC3 by P. UF1 in the pathologic conditions of NEC-like injury, in which the interplay between DCs and ILC3 implicated in the maintenance of epithelial barrier function (69-71) and CD $4^{+}$T cells (e.g., IL-10+ Th17 cells, Tregs) sustains functional intestinal homeostasis to potentially redirect deleterious signals (e.g., IL-1 $\beta$ ) (72) involved in neonatal immature immunity during inflammation (e.g., NEC). Together, these data indeed demonstrate the potency of P. UF1 in controlling the proinflammatory cells (e.g., Th17 cells) $(14,65)$ toward regulatory cells potentially resisting disease progression.

\section{Discussion}

Collectively, nutritional HBM and the bacterial species within (73, 74) not only govern both the development and the health of newborn infants $(75,76)$, but also affect optimal bacterial composition and related metabolites of the neonatal gut (77), thus influencing intestinal immunity and disease risk later in life (12). Yet mechanistic insights highlighting the critical role of HBM feeding are limited and require further investigation for understanding the underlying inputs promoting protective and regulated immune signaling that may be fine-tuned by gut microbiota and their induced metabolites. With this notion in mind, our studies demonstrate that HBM directs the fate of the bacterial composition of preterm infants and promotes the propagation of beneficial bacteria, including P.UF1, within preterm infants' gut microbiota; this critical trend was lacking in preterm infants fed formula. Importantly, this deficiency in the levels of beneficial bacteria undoubtedly contributes to uncontrolled inflammation that may potentially result in serious proinflammatory diseases such as NEC, wherein functional gut homeostasis is significantly impaired (14). This was evidenced by the fact that transfaunation of GF mice with FF fecal microbiota augmented proinflammation; however, addition of P. UF1 to this transfaunation mitigated the inflammatory responses and increased protective Th17 cells and Tregs. Such immune regulation was also shown in GF mice monoassociated with P. UF1, where, in addition to regulated innate and $\mathrm{T}$ cell responses, particularly those of Th17 cells and Tregs, critical regulatory metabolites (e.g., tryptophan, phenylalanine) (78) were also induced.

It was recently demonstrated that the differentiation of intestinal Th17 cells is highly dependent on their constant interaction with the gut microbiota, including SFB, a delicate association that determines the nature of immune responses in steady state and with disease (79). Likewise, we demonstrate that bacterial-dependent Th17 cell formation and its regulation is tightly intertwined with the bacterial DlaT gene product and is significantly fortified by functional Tregs and potentially key metabolites (e.g., tryptophan, kynurenine) (80). Importantly, the formation of protective DlaT-specific Th17 cells was robustly sustained during $L$. $m$ infection, a trend that was not observed using SFB-specific peptides expressed by $L$. $m$ that skewed these cells toward Th1 responses (35). Here, the nature of the bacterial species (34) may influence T cell plasticity to either differentiate into gut regulatory Th17 cells (e.g., P. UF1) or proinflammatory Th1 cell responses (e.g., SFB). 
A Isotype PBS P. UF1
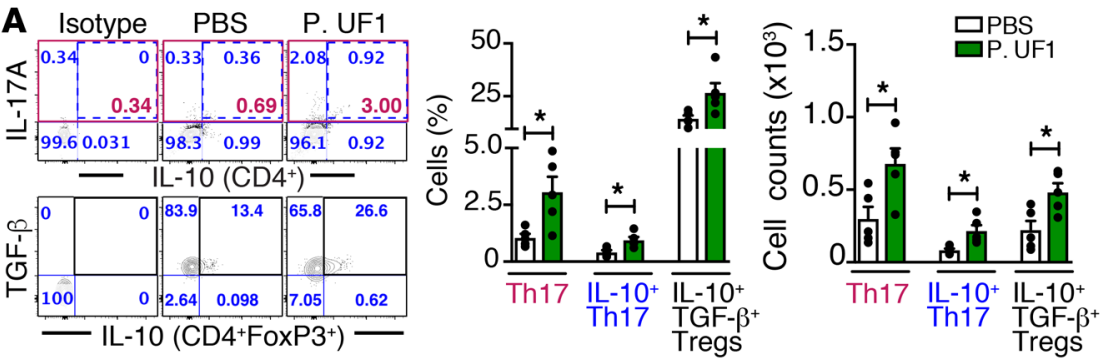

B $-\mathrm{NEC}$

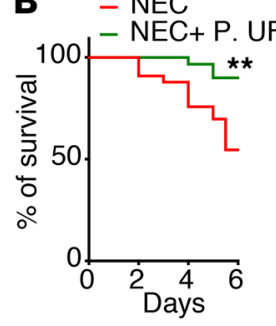

$\rightarrow$ NEC

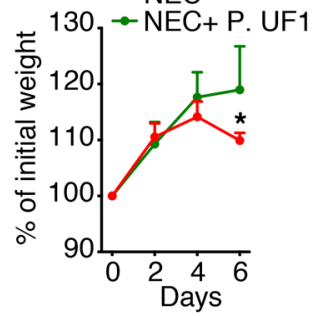

C

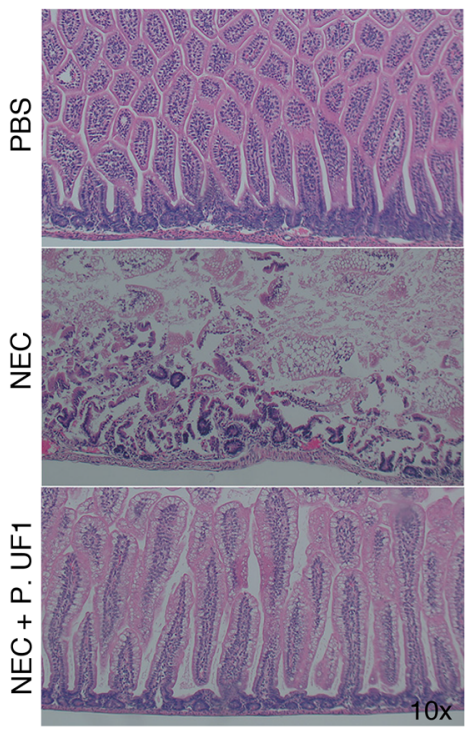

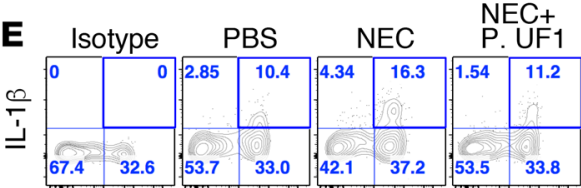
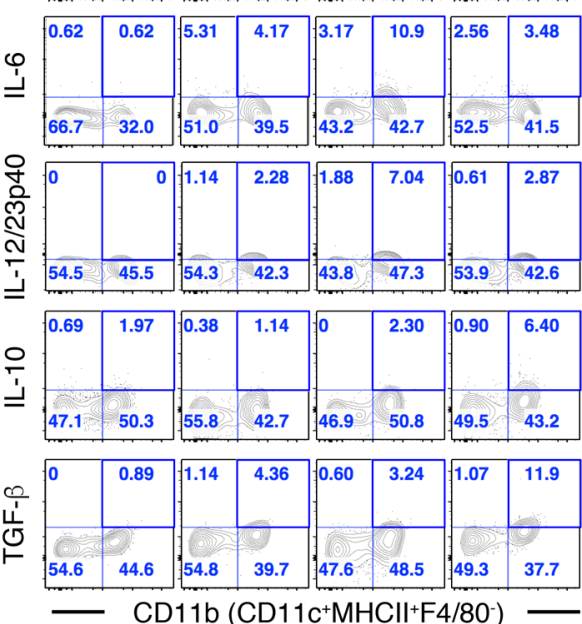

$\mathrm{CD} 11 \mathrm{~b}\left(\mathrm{CD} 11 \mathrm{C}^{+} \mathrm{MHCI}+\mathrm{F} 4 / 80^{-}\right)$
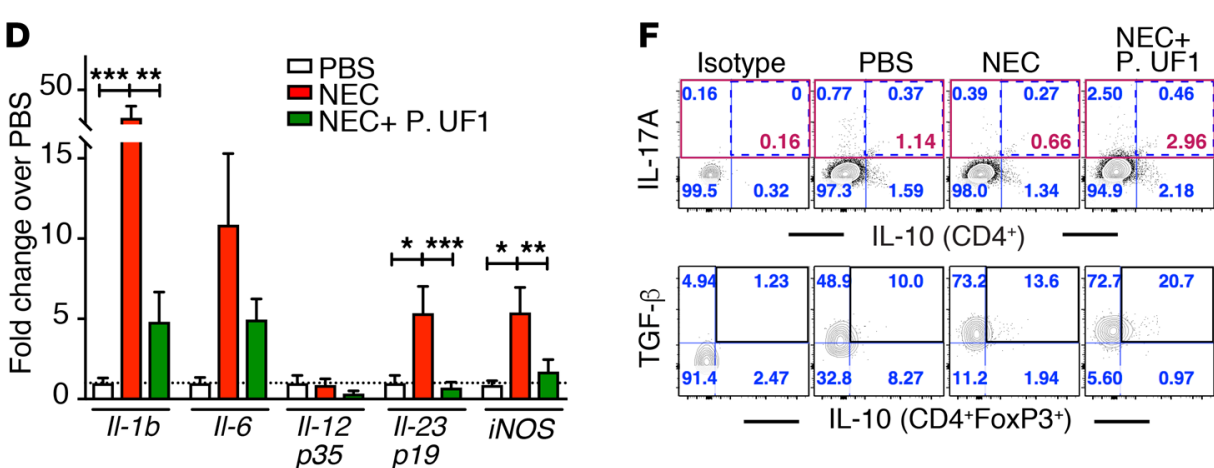

$\mathbf{F}$

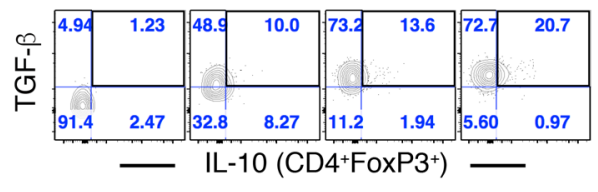

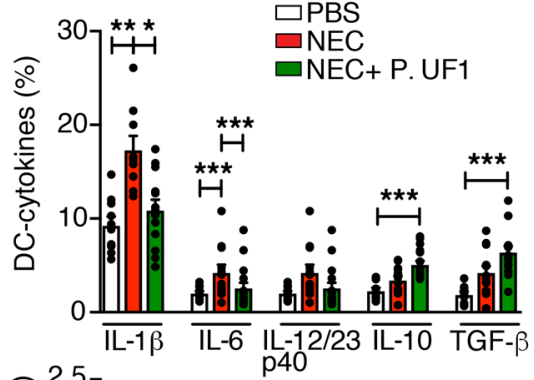

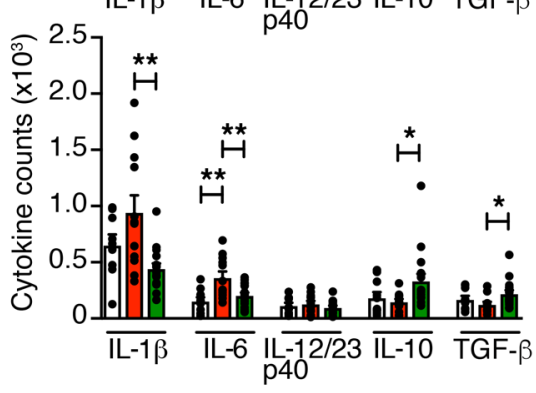

Figure 9. Mitigation of NEC-like injury by P. UF1 in newborn mice. (A) C57BL/6 pregnant dams were gavaged with P. UF1 or PBS twice/week during gestation. Five days after birth, newborn mice were sacrificed. Representative data of flow plots, percentages, and total cell counts of Th17 cells and IL-10+TGF- $\beta^{+}$Tregs in newborn mice. Each dot represents 3 pooled small intestinal tissues from each group of newborn mice. (B and C) Five days after birth, newborn mice of dams gavaged with P. UF1 or PBS were separated and subjected to NEC-like injury. Newborn mice gavaged with PBS or P. UF1 ( $10^{7}$ CFU/mouse) on days 1,3 , and 5 . Newborn mice were sacrificed 6 days later. Survival curve and weight incidence of the newborn mice subjected to NEC-like injury (B), and representative H\&E sections of the small intestine of control newborn mice (PBS) or mice subjected to NEC-like injury with or without P. UF1. (C) Original magnification, $\times 10$. (D) qRT-PCR demonstrating expression of proinflammatory genes in the small intestine of P. UF1-gavaged or untreated newborn mice subjected to NEC-like injury. Control newborn mice (PBS) with no NEC-like injury. (E and F) Representative data of flow plots, percentages, and total cell counts of IL-1 $\beta^{+}$, IL- $6^{+}, I L-12 / 23 p 40^{+}$, IL-10+ , and TGF- $\beta^{+}$DCs (E), IL-10+ Th17 cells, and IL-10+ TCF- $\beta^{+}$Tregs (F) in the indicated groups. Data are pooled from 3 experiments in steady state ( $n=15$ newborn mice/group, A) and for NEC-like injury (PBS, $n=10$ newborn mice; NEC, $n=12$ newborn mice; NEC + P. UF1, $n=17$ newborn mice, E) or 4 experiments (NEC, $n=33$ newborn mice; NEC + P. UF1, $n=30$ newborn mice, B). Error bars represent mean $\pm \mathrm{SEM}$. ${ }^{*} P<0.05 ;{ }^{* *} P<0.01 ;{ }^{* *} P<0.001 ;{ }^{* * *} P<0.0001,2$-tailed unpaired $t$ test $(\mathbf{A}$ and $\mathbf{B})$ or Kruskal-Wallis test $(\mathbf{D}-\mathbf{F})$. 
Further, P. UF1 not only regulated protective IFN $-\gamma^{+}$Th1 cells and IL-10+ ${ }^{+}$Th17 cells (81) and sustained IL-10 ${ }^{+}$Tregs to successfully eliminate $\triangle a c t A$ L. $m$ (82), but it also resisted microbial dysbiosis potentially induced by $L$. $m$ infection. P. UF1 also elicited critical metabolites (e.g., citrulline) involved in immune protection. In this regard, DlaT not only influences Th17 cells, but also directs the bacterial molecular machinery, as its deletion profoundly changes the bacterial transcriptome orchestrating metabolomic cycles, resulting in further inflammation deleterious to protection against pathogen infection. Yet, does IL-17 play a role against intracellular pathogens such as $L . m$ ? Classically protective responses against L. $m$ were considered to require IFN- $\gamma^{+}$Th1 cells (48). However, IFN- $\gamma$ deficiency does not exclude the activation of $\mathrm{CD}^{+} \mathrm{T}$ cells against this pathogen (83). Moreover, protective Th17 cells were induced against several intracellular pathogens, including Salmonella typhimurium (84) and L. $m$ (85), indicating that Th17 cells are not redundant against intracellular pathogens. Thus, synergistically induced Th1 and Th17 cell responses will swiftly resolve the infection and limit tissue damage (86). Here, we demonstrate this side of protective immunity directed by P. UF1 against $L . m$ infection, a model in which both Th1 and Th17 cells were highly regulated. Studies are required to further elucidate underlying mechanisms implicated in protective Th17 cells that may bridge protective responses to fortify Th1 cell activation against intracellular pathogens.

CLRs, particularly SIGNR1, expressed by phagocytes (e.g., DCs) are critical pattern-recognition receptors interacting with microbial carbohydrate recognition domains (CRDs) (87) to efficiently internalize and phagocytize microbes in concert with other molecules, including complement receptors (88), and to potentiate the oxidative responses to pathogens (e.g., Candida albicans) via dectin-1/SyK-dependent signaling (89). One can speculate that the signaling pathways of SIGNR1 involve regulated immune complexes (e.g., SOCS1) whose activation may be dependent on a mannosylated S-layer binding to SIGNR1 to control proinflammation in DCs that influence regulatory Th17 cells $(90,91)$. Here, we show that SIGNR1 signaling participates in DC regulation by activating Socs 1 to control proinflammatory signaling (e.g., IL-1 $\beta$ ) (92), promoting IL-10 ${ }^{+}$Th17 cells by suppressing STAT1 signaling, thus limiting IFN- $\gamma$ signaling in these cells (93). Disruption of SIGNR1 signaling (e.g., SOCS1) results in augmented pathogenic conditions, leading to further disequilibrium and lack of a functional homeostasis required for resolving $L . m$ infection. Accordingly, future investigations are warranted to assess the impact of signals directed by SIGNR1 interaction with P. UF1 that mediate regulation during inflammatory conditions and whether mannose-containing S-layer constituents of P. UF1, including lipomannan synthesized by glycolipid pathways involving mannosyltransferases PimA and MptA (94), initiate regulatory signals (SOCS1) in intestinal DCs that skew pathogen-induced inflammation toward regulation.

Although the underlying pathological mechanisms involved in NEC are currently elusive and what is commonly termed NEC in human infants likely represents several different mechanistic pathways, induced pathogenic inflammation implicated in disease manifestation is of significant relevance $(6,95)$. To investigate whether P. UF1 ameliorates pathogenic inflammation induced in newborn mice, an experimental NEC-like injury was employed whereby severe epithelial damage can be observed $(63,64)$. In this murine model, P. UF1 regulated deleterious signals (e.g., iNOS, IL-1 $\beta$ ) involved in the pathogenesis of NEC-like injury by controlling DCs and sustaining IL-10 ${ }^{+}$Tregs to potentially regulate $\mathrm{IL}-10^{+}$Th17 cells $(81,96)$ and IL-17A $\mathrm{AL}^{+}-22^{+}$ILC3, all of which render a condition to oppose the manifestation of NEC-like injury in newborn mice. Such regulatory conditions were also established by feeding P. UF1 to pregnant dams compared with feeding it to control newborn mice after birth. In this permutation, P. UF1 coordinates critical signals in DCs to produce protective IL-10 (97) and TGF- $\beta$; a trend that can potentially promote IL-10+ ${ }^{+}$Tregs and their mobilization against ensuing pathogenic inflammation, as seen in newborn mice subjected to NEC-like injury. These encouraging data may pave the way to further elucidate critical transcriptic and metabolic factors embedded within the P. UF1 genomic machinery and their relevance in the control of pathogenic inflammation occurring with NEC in preterm infants and potential immunotherapeutic translation in the clinic setting in the near future.

In summary, the current studies elucidate mechanisms involved in the attenuation of inflammatory responses affecting cellular homeostasis via the interaction of P. UF1 with SIGNR1. This results in the regulation of proinflammatory signals (e.g., IL-1 $\beta$ ) promoting functional $\mathrm{T}$ cell homeostasis (regulatory Th17 cells) to efficiently clear pathogens and to limit disease progression. Errors in these molecular mechanisms mobilize pathologic signals, resulting in destruction of intestinal homeostasis, leading to devastating proinflammatory diseases, including NEC. Thus, the current investigation may address some of the knowledge gap related to HBM feeding and its impact on the signaling complexes that directly reduce intestinal inflammation as well as distal tissue damage associated with intestinal disorders (e.g., NEC). The impact of P. UF1-induced metabolites on gut homeostasis remains to be determined in order to draw a clear conclusion on the critical metabolites involved in regulated immunity against pathogens and disease. Independent of such considerations, our data distinctly support the notion that HBM, the bacteria within (e.g., P. UF1), and their gene products (e.g., DlaT) critically regulate the proinflammatory environment potentially implicated in NEC affecting preterm infants. Accordingly, candidates for modulators of the local response with translational importance would be beneficial bacteria such as P. UF1. Future strategies to restore the balance of regulatory lymphocytes may provide novel approaches to dampen inflammation and to prevent inflammatory disease progression such as that seen with NEC.

\section{Methods}

Human fecal sample collection. Preterm infants with gestational ages less than or equal to 32 completed weeks and birth weights less than or equal to $1,800 \mathrm{~g}$ were eligible for our study. Infants with major congenital anomalies or malformations were excluded. Infants from 3 University of Florida-affiliated hospitals who met the inclusion criteria were enrolled in the study shortly after birth. Stool samples were collected weekly from the study infants starting with meconium and continued until discharge. The samples were immediately stored at $-80^{\circ} \mathrm{C}$. The analyzed selected samples were derived from control infants who did 
not develop NEC or culture positive for sepsis (Supplemental Table 1). Two groups, each with 20 subjects, were selected and either received an HBMF diet or an FF diet. For each group, subject samples from 2 different time points were analyzed: the first group of samples was collected at $13 \pm 2$ to 3 days after birth with $11 \pm 3$ days of feeding, and the second group of samples was collected at $21 \pm 3$ days after birth with $19 \pm 4$ days of feeding.

Animals. C57BL/6, B6.PL-Thy1a/CyJ, B6.129(Cg)-Foxp3 $3^{\text {tm3(DTR/GFP)Ayr } / \mathrm{J}}$

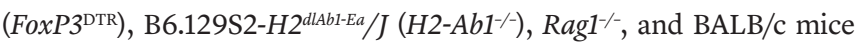
were obtained from Jackson Laboratory. Signr1-/- mice and B6.129P2(Cg)$\operatorname{Rorc}^{\text {tm2Litt }} / \mathrm{J}\left(\operatorname{Rorc}(\gamma \mathrm{t})^{\mathrm{GFP} / \mathrm{GFP}}\right)$ mice were provided by Huang Shau-Ku (Johns Hopkins University School of Medicine, Baltimore, Maryland, USA) and Liang Zhou (University of Florida). C57BL/6 GF mice were provided by the Animal Care Facility of the University of Florida and maintained there until the experiments were performed. Mice (5 days old or 6 to 8 weeks old) were maintained under specific pathogen-free, Helicobacter-free conditions.

First draft genome analyses of $P$. UF1. Fecal samples from HBMF preterm infants $(100 \mathrm{mg}$ ) were dissolved in sterile lectin-binding buffer (98), and biotinylated Concanavalin A (ConA, $100 \mu \mathrm{g}$ ) (Vector Laboratories) was added to the suspension. The fecal contents were incubated at $4^{\circ} \mathrm{C}$ for 3 hours and subsequently washed with lectinbinding buffer 3 times. The streptavidin-ferrous beads (Thermo Fisher Scientific) were then added to the washed fecal contents, allowing the ConA-bacteria complex to bind to the beads. The ferrous beads were washed 6 times with lectin-binding buffer in the presence of a magnet. The enriched bacteria, via bead complex, were spread over several MRS-lactate agar plates (MRS agar with 1\% sodium lactate; $\mathrm{w} / \mathrm{v}$ ) to grow the separated bacteria for 3 days. Bacterial DNA of the colonies was isolated, and bacterial identity was determined by the amplification of 16S rRNA gene and sequencing analyses. One of the isolates was designated as P. UF1. Genomic DNA of the P. UF1 was isolated, purified, and subjected to PACBIO RS II for whole genome sequencing (99). The sequenced contigs by PACBIO RS II were assembled into 1 unique contig using SMRT Portal (Pacific Biosciences), which was then compared with known sequences of $P$. freudenreichii subsp. shermanii CIRM-BIA1 and of $P$. freudenreichii subsp. freudenreichii DSM20271 ${ }^{\mathrm{T}}$. A distinguishable prophage identified by PHAST (Phage Search Tool) was also shown in the comparison to demonstrate the key bacterial strain differences and the missing gaps visualized in a circular genome map. The circular map was generated using Blast Ring Image Generator (BRIG) (100). The genome of the P. UF1 was annotated and combined using Rapid Annotation Server (RAST), KEGG Orthology And Links Annotation (KOALA) (101), and Rapid Prokaryotic Genome Annotation (PROKKA) (102). The subsystem categories were summarized and visualized by using RAST. The genome sequence of P. UF1 was submitted to the NCBI's Genome database (CP018002). The P. UF1 isogenic strains were constructed as described in Supplemental Experimental Procedures, using primers presented in Supplemental Table 2.

Ultra-high resolution metabolomic analyses. Mouse fecal samples and P. UF1 bacterial cultures were collected and analyzed at the Department of Medicine Clinical Biomarkers Laboratory at Emory University $(27,103)$, as described in Supplemental Experimental Procedures.

Experimental NEC-like injury. C57BL/6 female (dam) mice were gavaged during pregnancy with P. UF1 $\left(10^{9} \mathrm{CFU} / \mathrm{dam}\right)$ or PBS twice weekly. On day 5 , newborn mice were subjected to experimental
NEC-like injury. Briefly, the cecal contents of 3 healthy adult mice (6 weeks old) were cultured overnight at $37^{\circ} \mathrm{C}$ in regular bacterial culture media (1:1) (LB Broth Base, GIBCO, Invitrogen). The CFU of stock mixture bacteria was then determined. Multiple aliquots were frozen at $-80^{\circ} \mathrm{C}$. The day prior to inducing NEC-like injury, an aliquot was cultured for 16 hours at $37^{\circ} \mathrm{C}$ to gavage newborn mice with $10^{7} \mathrm{CFU}$-cultured bacteria. Newborn mice were gavaged twice daily with $10^{7} \mathrm{CFU}$ of a standardized adult commensal bacterial mixture (104) for 4 days. Newborn mice were gavaged 6 times daily with formula (Similac Advance infant formula, Abbott Nutrition; Esbilac canine milk replacer, PetAg; at the ratio 2:1). Newborn mice were exposed to episodes of hypoxia (300 seconds in 95\% $\mathrm{N}_{2}, 5 \% \mathrm{O}_{2}$ ) twice daily for 4 days. Newborn mice were observed closely for clinical signs, including severe abdominal distension, apnea, cyanosis, lethargy, and weight loss. Newborn mice from P. UF1-gavaged dams were also gavaged with P. UF1 $\left(10^{7} \mathrm{CFU} /\right.$ mouse) during experimental procedures on days 1,3 , and 5 . Newborn mice were sacrificed 6 days later. PBS-gavaged newborn mice that remained with their mothers were used as controls.

Histopathology. The severity of intestinal injury was determined by histopathology. The entire small intestine was fixed, sectioned, and stained with H\&E. Stained sections were blindly evaluated by a boarded veterinary pathologist.

Statistics. Statistical analyses were performed using GraphPad Prism (version 6.0 for Mac OS X). Mean and SEM values and statistical significance between 2 variables were determined by 2 -tailed unpaired $t$ tests. Normality was tested using the Shapiro-Wilk normality test. Where the groups follow a Gaussian distribution, parametric analyses were performed (2-tailed unpaired $t$ test for 2 variables or 1-way ANOVA followed by Tukey's post test for 3 or more variables). Where the groups did not follow a Gaussian distribution, nonparametric analyses were performed (Mann-Whitney $U$ test for 2 variables or Kruskal-Wallis test followed by Dunn's post test for 3 or more variables). Differences were considered to be significant at $P<0.05$. For microbiota analyses, the Kruskal-Wallis test was used in LEfSe to perform statistical testing for microbiota compositions. Differences were considered to be significant at $P$ values of less than 0.05 , and only features with an LDA score of more than 2 were shown. For tests in differential gene expression analyses, the negative binomial (NB) model was applied as the statistical model to compute $P$ values, followed by the Benjamini-Hochberg procedure to calculate $q$ values (105). For metabolomic analyses, the differential expression of fecal metabolites among different groups was determined with $t$ tests or ANOVA tests using Python, and pathway and network analyses were performed using Mummichog, a set of algorithms specifically designed for high-throughput metabolomics (106).

Study approval. Fecal samples were collected after informed written consent from a parent and approval by the IRB (no. 386-2008) at the University of Florida. All animal studies were approved by the IACUC of the University of Florida under the following protocol numbers: 201406559, 201408484, and 201609388. Mice were maintained in accordance with the Animal Welfare Act and the Public Health Policy on Humane Care.

\section{Author contributions}

MM directed and designed the cellular and molecular experiments, which were executed by NC, YG, BS, MG, MZ, JLO, and FA. NC, MZ, 
YG, and BS performed the mouse work. YG, MG, and FA performed the molecular studies, gene deletion and complementation, transcriptomic studies, and generation of $\triangle a c t A L . m$ and $\triangle a c t A L . m^{3 p e p}$. SL and DJ performed, analyzed, and directed metabolomic studies. SL, KL, and MG conducted all bioinformatic work for metabolic data analyses. WGF analyzed the genome of P. UF1. MG and YG performed experiments involving microbiota, transcriptomic analyses, and P. UF1 genome-wide analyses. JN collected and provided the fecal samples. NC, YG, MG, BS, JLO, JN, FA, SL, DPJ, and MM analyzed and interpreted the data. MM wrote the manuscript.

\section{Acknowledgments}

This work was supported by NIH R01 DK109560 (to MM), the NIH/NCRR Clinical and Translational Science Award (to MM), and Gatorade Trust Funds Florida (to MM). We thank Melissa N. Valletti and Max R. Van Belkum for excellent technical assistance.

Address correspondence to: Mansour Mohamadzadeh, Department of Infectious Diseases \& Immunology, University of Florida, 2015 SW 16th Avenue, Building 1017, Gainesville, Florida 32608, USA. Phone: 352.294.4117; Email:m.zadeh@ufl.edu.
1. Goldenberg RL, Culhane JF, Iams JD, Romero R. Epidemiology and causes of preterm birth. Lancet. 2008;371(9606):75-84.

2. Romero R, Espinoza J, Gonçalves LF, Kusanovic JP, Friel L, Hassan S. The role of inflammation and infection in preterm birth. Semin Reprod Med. 2007;25(1):21-39.

3. Groer MW, Luciano AA, Dishaw LJ, Ashmeade TL, Miller E, Gilbert JA. Development of the preterm infant gut microbiome: a research priority. Microbiome. 2014;2:38.

4. Lim JC, Golden JM, Ford HR. Pathogenesis of neonatal necrotizing enterocolitis. Pediatr Surg Int. 2015;31(6):509-518.

5. Besner GE. A pain in the NEC: research challenges and opportunities. J Pediatr Surg. 2015;50(1):23-29.

6. Neu J, Walker WA. Necrotizing enterocolitis. N Engl JMed. 2011;364(3):255-264.

7. Donovan SM, Wang M, Li M, Friedberg I, Schwartz SL, Chapkin RS. Host-microbe interactions in the neonatal intestine: role of human milk oligosaccharides. Adv Nutr. 2012;3(3):450S-455S.

8. Murgas Torrazza R, Neu J. The developing intestinal microbiome and its relationship to health and disease in the neonate. J Perinatol. 2011;31 Suppl 1:S29-S34.

9. Morelli L. Postnatal development of intestinal microflora as influenced by infant nutrition. J Nutr. 2008;138(9):1791S-1795S.

10. Bode L. Human milk oligosaccharides: prebiotics and beyond. Nutr Rev. 2009;67 Suppl 2:S183-S191.

11. David LA, et al. Diet rapidly and reproducibly alters the human gut microbiome. Nature. 2014;505(7484):559-563.

12. Urbaniak C, et al. Effect of chemotherapy on the microbiota and metabolome of human milk, a case report. Microbiome. 2014;2:24.

13. Littman DR, Rudensky AY. Th17 and regulatory T cells in mediating and restraining inflammation. Cell. 2010;140(6):845-858.

14. Egan CE, et al. Toll-like receptor 4-mediated lymphocyte influx induces neonatal necrotizing enterocolitis. JClin Invest. 2016;126(2):495-508.

15. Hibbing ME, Fuqua C, Parsek MR, Peterson SB. Bacterial competition: surviving and thriving in the microbial jungle. Nat Rev Microbiol. 2010;8(1):15-25.

16. Bäumler AJ, Sperandio V. Interactions between the microbiota and pathogenic bacteria in the gut. Nature. 2016;535(7610):85-93.

17. Grimm V, Radulovic K, Riedel CU. Colonization of C57BL/ 6 mice by a potential probiotic bifidobacterium bifidum strain under germ- free and specific pathogen-free conditions and during experimental colitis. PLOS ONE. 2015;10(10):e0139935.

18. Kim YG, et al. Neonatal acquisition of Clostridia species protects against colonization by bacterial pathogens. Science. 2017;356(6335):315-319.

19. Ridaura VK, et al. Gut microbiota from twins discordant for obesity modulate metabolism in mice. Science. 2013;341(6150):1241214.

20. Chow J, Tang H, Mazmanian SK. Pathobionts of the gastrointestinal microbiota and inflammatory disease. Curr Opin Immunol. 2011;23(4):473-480.

21. Kamada N, Chen GY, Inohara N, Núñez G. Control of pathogens and pathobionts by the gut microbiota. Nat Immunol. 2013;14(7):685-690.

22. Kamada N, Seo SU, Chen GY, Núñez G. Role of the gut microbiota in immunity and inflammatory disease. Nat Rev Immunol. 2013;13(5):321-335.

23. Coccia $\mathrm{M}$, et al. IL-1 $\beta$ mediates chronic intestinal inflammation by promoting the accumulation of IL-17A secreting innate lymphoid cells and CD4(+) Th17 cells. JExp Med. 2012;209(9):1595-1609.

24. Negrete A, Majdalani N, Phue JN, Shiloach J. Reducing acetate excretion from E. coli K-12 by over-expressing the small RNA SgrS. $N$ Biotechnol. 2013;30(2):269-273.

25. Barnes MJ, Powrie F. Regulatory T cells reinforce intestinal homeostasis. Immunity. 2009;31(3):401-411.

26. Veldhoen M, Hirota K, Christensen J, O'Garra A, Stockinger B. Natural agonists for aryl hydrocarbon receptor in culture medium are essential for optimal differentiation of Th17 T cells. J Exp Med. 2009;206(1):43-49.

27. Li S, et al. Predicting network activity from high throughput metabolomics. PLoS Comput Biol. 2013;9(7):e1003123.

28. Libert C, Dejager L. How steroids steer T cells. Cell Rep. 2014;7(4):938-939.

29. Ulvik A, Midttun Ø, Pedersen ER, Nygård O, Ueland PM. Association of plasma B-6 vitamers with systemic markers of inflammation before and after pyridoxine treatment in patients with stable angina pectoris. Am J Clin Nutr. 2012;95(5):1072-1078.

30. Murakami K, Korbsrisate S, Asahara N, Hashimoto Y, Murooka Y. Cloning and characterization of the glutamate 1-semialdehyde aminomutase gene from Xanthomonas campestris pv. phaseoli. Appl Microbiol Biotechnol. 1993;38(4):502-506.

31. Lightfoot YL, et al. SIGNR3-dependent immune regulation by Lactobacillus acidophilus surface layer protein A in colitis. $Е M B O J$. 2015;34(7):881-895.
32. Lathrop SK, et al. Peripheral education of the immune system by colonic commensal microbiota. Nature. 2011;478(7368):250-254.

33. Sefik E, et al. Mucosal immunology. individual intestinal symbionts induce a distinct population of $\mathrm{ROR} \gamma^{+}$regulatory $\mathrm{T}$ cells. Science. 2015;349(6251):993-997.

34. Geva-Zatorsky N, et al. Mining the human gut microbiota for immunomodulatory organisms. Cell. 2017;168(5):928-943.e11.

35. Yang Y, et al. Focused specificity of intestinal TH17 cells towards commensal bacterial antigens. Nature. 2014;510(7503):152-156.

36. Ivanov II, et al. Induction of intestinal Th17 cells by segmented filamentous bacteria. Cell. 2009;139(3):485-498.

37. Veldhoen M, Hocking RJ, Atkins CJ, Locksley RM, Stockinger B. TGFbeta in the context of an inflammatory cytokine milieu supports de novo differentiation of IL-17-producing T cells. Immunity. 2006;24(2):179-189.

38. Zhou ZH, McCarthy DB, O'Connor CM, Reed LJ, Stoops JK. The remarkable structural and functional organization of the eukaryotic pyruvate dehydrogenase complexes. Proc Natl Acad Sci U S A. 2001;98(26):14802-14807.

39. Goto Y, et al. Segmented filamentous bacteria antigens presented by intestinal dendritic cells drive mucosal Th17 cell differentiation. Immunity. 2014;40(4):594-607.

40. Mucida D, Park Y, Cheroutre H. From the diet to the nucleus: vitamin A and TGF-beta join efforts at the mucosal interface of the intestine. Semin Immunol. 2009;21(1):14-21.

41. ten Broeke T, Wubbolts R, Stoorvogel W. MHC class II antigen presentation by dendritic cells regulated through endosomal sorting. Cold Spring Harb Perspect Biol. 2013;5(12):a016873.

42. Mohamadzadeh M, et al. Regulation of induced colonic inflammation by Lactobacillus acidophilus deficient in lipoteichoic acid. Proc Natl Acad Sci U S A. 2011;108 Suppl 1:4623-4630.

43. Mombaerts P, Iacomini J, Johnson RS, Herrup K, Tonegawa S, Papaioannou VE. RAG-1-deficient mice have no mature B and T lymphocytes. Cell. 1992;68(5):869-877.

44. Ge Y, Zhao N, Hu X, Shi T, Cai Q, Yuan Z. A novel transcriptional activator, tubX, is required for the stability of Bacillus sphaericus mosquitocidal plasmid pBsph. J Bacteriol. 2014;196(24):4304-4314.

45. Liang SC, et al. Interleukin (IL)-22 and IL-17 are coexpressed by Th17 cells and cooperatively enhance expression of antimicrobial peptides. 
JExp Med.2006;203(10):2271-2279.

46. Gaboriau-Routhiau V, et al. The key role of segmented filamentous bacteria in the coordinated maturation of gut helper $\mathrm{T}$ cell responses. Immunity. 2009;31(4):677-689.

47. Chung $\mathrm{H}$, et al. Gut immune maturation depends on colonization with a host-specific microbiota. Cell. 2012;149(7):1578-1593.

48. Hsieh CS, Macatonia SE, Tripp CS, Wolf SF, O'Garra A, Murphy KM. Development of TH1 CD4+ T cells through IL-12 produced by Listeria-induced macrophages. Science. 1993;260(5107):547-549.

49. Kocks C, Gouin E, Tabouret M, Berche P, Ohayon H, Cossart P. L. monocytogenes-induced actin assembly requires the actA gene product, a surface protein. Cell. 1992;68(3):521-531.

50. Zhou L, Littman DR. Transcriptional regulatory networks in Th17 cell differentiation. Curr Opin Immunol. 2009;21(2):146-152.

51. Benson A, et al. Microbial infection-induced expansion of effector $\mathrm{T}$ cells overcomes the suppressive effects of regulatory $\mathrm{T}$ cells via an IL-2 deprivation mechanism. J Immunol. 2012;188(2):800-810.

52. Ravindran $\mathrm{R}$, et al. Vaccine activation of the nutrient sensor GCN2 in dendritic cells enhances antigen presentation. Science. 2014;343(6168):313-317.

53. Kim JM, Rasmussen JP, Rudensky AY. Regulatory $\mathrm{T}$ cells prevent catastrophic autoimmunity throughout the lifespan of mice. Nat Immunol. 2007;8(2):191-197.

54 . Wang Z, et al. Regulatory $\mathrm{T}$ cells promote a protective Th17-associated immune response to intestinal bacterial infection with C. rodentium. Mucosal Immunol. 2014;7(6):1290-1301.

55. Geijtenbeek TB, van Vliet SJ, Engering A, 't Hart $B A$, van Kooyk Y. Self- and nonself-recognition by C-type lectins on dendritic cells. Annu Rev Immunol. 2004;22:33-54.

56. Lightfoot YL, et al. SIGNR3-dependent immune regulation by Lactobacillus acidophilus surface layer protein A in colitis. $E M B O J$. 2015;34(7):881-895.

57. Srivastava V, et al. Toll-like receptor 2 and DCSIGNR1 differentially regulate suppressors of cytokine signaling 1 in dendritic cells during Mycobacterium tuberculosis infection. J Biol Chem. 2009;284(38):25532-25541.

58. den Dunnen J, Gringhuis SI, Geijtenbeek TB. Dusting the sugar fingerprint: C-type lectin signaling in adaptive immunity. Immunol Lett. 2010;128(1):12-16.

59. Yang J, et al. Pathological axonal death through a MAPK cascade that triggers a local energy deficit. Cell. 2015;160(1-2):161-176.

60. Gomez de Agüero M, et al. The maternal microbiota drives early postnatal innate immune development. Science. 2016;351(6279):1296-1302.

61. Torrazza RM, Neu J. The altered gut microbiome and necrotizing enterocolitis. Clin Perinatol. 2013;40(1):93-108.

62. Neu J. The microbiome during pregnancy and early postnatal life. Semin Fetal Neonatal Med. 2016;21(6):373-379.

63. Egan CE, et al. Toll-like receptor 4-mediated lymphocyte influx induces neonatal necrotizing enterocolitis. J Clin Invest. 2016;126(2):495-508.

64. Tian R, et al. Characterization of a necrotizing enterocolitis model in newborn mice. Int JClin Exp Med.2010;3(4):293-302.

65. Underwood MA, et al. Bifidobacterium longum subsp. infantis in experimental necrotizing enterocolitis: alterations in inflammation, innate immune response, and the microbiota. Pediatr Res. 2014;76(4):326-333.

66. Chen L, et al. IL-23 activates innate lymphoid cells to promote neonatal intestinal pathology. Mucosal Immunol. 2015;8(2):390-402.

67. Sonnenberg GF, Artis D. Innate lymphoid cells in the initiation, regulation and resolution of inflammation. Nat Med. 2015;21(7):698-708.

68. Hepworth MR, et al. Innate lymphoid cells regulate CD4+ T-cell responses to intestinal commensal bacteria. Nature. 2013;498(7452):113-117.

69. Rankin LC, et al. Complementarity and redundancy of IL-22-producing innate lymphoid cells. Nat Immunol. 2016;17(2):179-186.

70. Sonnenberg GF, Fouser LA, Artis D. Border patrol: regulation of immunity, inflammation and tissue homeostasis at barrier surfaces by IL-22 Nat Immunol. 2011;12(5):383-390.

71. Sugimoto K, et al. IL-22 ameliorates intestinal inflammation in a mouse model of ulcerative colitis. J Clin Invest. 2008;118(2):534-544.

72. Coccia $\mathrm{M}$, et al. IL-1 $\beta$ mediates chronic intestinal inflammation by promoting the accumulation of IL-17A secreting innate lymphoid cells and CD4(+) Th17 cells. JExp Med. 2012;209(9):1595-1609.

73. Díaz-Ropero MP, et al. Two Lactobacillus strains, isolated from breast milk, differently modulate the immune response. J Appl Microbiol. 2007;102(2):337-343.

74. Lara-Villoslada F, Olivares M, Sierra S, Rodríguez JM, Boza J, Xaus J. Beneficial effects of probiotic bacteria isolated from breast milk. Br J Nutr. 2007;98 Suppl 1:S96-100.

75. Daniels MC, Adair LS. Breast-feeding influences cognitive development in Filipino children. J Nutr. 2005;135(11):2589-2595.

76. German JB, Dillard CJ, Ward RE. Bioactive components in milk. Curr Opin Clin Nutr Metab Care. 2002;5(6):653-658.

77. Zivkovic AM, German JB, Lebrilla CB, Mills DA. Human milk glycobiome and its impact on the infant gastrointestinal microbiota. Proc Natl Acad Sci U S A. 2011;108 Suppl 1:4653-4658.

78. Sikalidis AK. Amino acids and immune response: a role for cysteine, glutamine, phenylalanine, tryptophan and arginine in T-cell function and cancer? Pathol Oncol Res. 2015;21(1):9-17.

79. Ivanov II, et al. The orphan nuclear receptor RORgammat directs the differentiation program of proinflammatory IL-17+ T helper cells. Cell. 2006;126(6):1121-1133.

80. Romani L, Zelante T, De Luca A, Fallarino F, Puccetti P. IL-17 and therapeutic kynurenines in pathogenic inflammation to fungi. J Immunol. 2008;180(8):5157-5162.

81. McGeachy MJ, et al. TGF-beta and IL-6 drive the production of IL-17 and IL-10 by T cells and restrain T(H)-17 cell-mediated pathology. Nat Immunol. 2007;8(12):1390-1397.

82. Khader SA, Gopal R. IL-17 in protective immunity to intracellular pathogens. Virulence.
2010;1(5):423-427.

83. Harty JT, Bevan MJ. Specific immunity to Listeria monocytogenes in the absence of IFN gamma. Immunity. 1995;3(1):109-117.

84. Thiemann S, et al. Enhancement of IFN $\gamma$ production by distinct commensals ameliorates salmonella-induced disease. Cell Host Microbe. 2017;21(6):682-694.e5.

85. Chung Y, et al. Epstein Barr virus-induced 3 (EBI3) together with IL-12 negatively regulates $\mathrm{T}$ helper 17-mediated immunity to Listeria monocytogenes infection. PLoS Pathog. 2013;9(9):e1003628.

86. Veldhoen M. Interleukin 17 is a chief orchestrator of immunity. Nat Immunol. 2017;18(6):612-621.

87. Gringhuis SI, den Dunnen J, Litjens M, van der Vlist M, Geijtenbeek TB. Carbohydrate-specific signaling through the DC-SIGN signalosome tailors immunity to Mycobacterium tuberculosis, HIV-1 and Helicobacter pylori. Nat Immunol. 2009;10(10):1081-1088.

88. Kang YS, et al. A dominant complement fixation pathway for pneumococcal polysaccharides initiated by SIGN-R1 interacting with C1q. Cell. 2006;125(1):47-58.

89. Takahara K, Tokieda S, Nagaoka K, Takeda T, Kimura Y, Inaba K. C-type lectin SIGNR1 enhances cellular oxidative burst response against C. albicans in cooperation with Dectin-1. Eur J Immunol. 2011;41(5):1435-1444.

90. Esplugues E, et al. Control of TH17 cells occurs in the small intestine. Nature. 2011;475(7357):514-518.

91. Ohnmacht C, Marques R, Presley L, Sawa S, Lochner M, Eberl G. Intestinal microbiota, evolution of the immune system and the bad reputation of pro-inflammatory immunity. Cell Microbiol. 2011;13(5):653-659.

92. Ha YJ, et al. SOCS1 suppresses IL-1 $\beta$-induced $\mathrm{C} / \mathrm{EBP} \beta$ expression via transcriptional regulation in human chondrocytes. Exp Mol Med 2016;48:e241.

93. Dalpke AH, Opper S, Zimmermann S, Heeg K. Suppressors of cytokine signaling (SOCS)- 1 and SOCS- 3 are induced by CpG-DNA and modulate cytokine responses in APCs. JImmunol. 2001;166(12):7082-7089.

94. Tanne A, et al. A murine DC-SIGN homologue contributes to early host defense against Mycobacterium tuberculosis. J Exp Med. 2009;206(10):2205-2220.

95. Niño DF, Sodhi CP, Hackam DJ. Necrotizing enterocolitis: new insights into pathogenesis and mechanisms. Nat Rev Gastroenterol Hepatol. 2016;13(10):590-600.

96. Torchinsky MB, Garaude J, Martin AP, Blander JM. Innate immune recognition of infected apoptotic cells directs $\mathrm{T}(\mathrm{H}) 17$ cell differentiation. Nature. 2009;458(7234):78-82.

97. Emami CN, et al. Role of interleukin-10 in the pathogenesis of necrotizing enterocolitis. Am J Surg. 2012;203(4):428-435.

98. Eriksson M, Johannssen T, von Smolinski D, Gruber AD, Seeberger PH, Lepenies B. The C-type lectin receptor SIGNR3 binds to fungi present in commensal microbiota and influences immune regulation in experimental colitis. Front Immunol. 2013;4:196.

99. Kim KE, et al. Long-read, whole-genome shotgun sequence data for five model organisms. Sci 
Data. 2014;1:140045.

100.Alikhan NF, Petty NK, Ben Zakour NL, Beatson SA. BLAST Ring Image Generator (BRIG): simple prokaryote genome comparisons. BMC Genomics. 2011;12:402.

101. Kanehisa M, Sato Y, Morishima K. BlastKOALA and GhostKOALA: KEGG tools for functional characterization of genome and metagenome sequences. J Mol Biol. 2016;428(4):726-731. 102. Seemann T. Prokka: rapid prokaryotic genome annotation. Bioinformatics. 2014;30(14):2068-2069.

103. Yu T, Park Y, Li S, Jones DP. Hybrid feature detection and information accumulation using highresolution LC-MS metabolomics data. J Proteome Res. 2013;12(3):1419-1427.

104. Tian R, et al. Characterization of a necrotizing enterocolitis model in newborn mice. Int JClin Exp Med. 2010;3(4):293-302.

105. McClure R, et al. Computational analysis of bacterial RNA-Seq data. Nucleic Acids Res. 2013;41(14):e140.

106.Li S, et al. Predicting network activity from high throughput metabolomics. PLoS Comput Biol. 2013;9(7):e1003123. 